\title{
Notes on conformal invariance of gauge fields
}

\author{
Glenn Barnich \\ Physique Théorique et Mathématique \\ Université Libre de Bruxelles and International Solvay Institutes \\ Campus Plaine C.P. 231, B-1050 Bruxelles, Belgium \\ Xavier Bekaert \\ Laboratoire de Mathématiques et Physique Théorique \\ Unité Mixte de Recherche 7350 du CNRS \\ Fédération de Recherche 2964 Denis Poisson \\ Université François Rabelais \\ Parc de Grandmont, 37200 Tours, France \\ Maxim Grigoriev \\ I.E. Tamm Department of Theoretical Physics \\ P.N. Lebedev Physical Institute \\ Leninsky prospect 53, 119991 Moscow, Russia
}

\begin{abstract}
In Lagrangian gauge systems, the vector space of global reducibility parameters forms a module under the Lie algebra of symmetries of the action. Since the classification of global reducibility parameters is generically easier than the classification of symmetries of the action, this fact can be used to constrain the latter when knowing the former. We apply this strategy and its generalization for the non-Lagrangian setting to the problem of conformal symmetry of various free higher spin gauge fields. This scheme allows one to show that, in terms of potentials, massless higher spin gauge fields in Minkowski space and partially-massless fields in (A)dS space are not conformal for spin strictly greater than one, while in terms of curvatures, maximal-depth partially-massless fields in four dimensions are also not conformal, unlike the closely related, but less constrained, maximal-depth Fradkin-Tseytlin fields.
\end{abstract}




\section{Contents}

1 Generalities 3

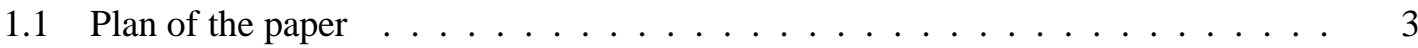

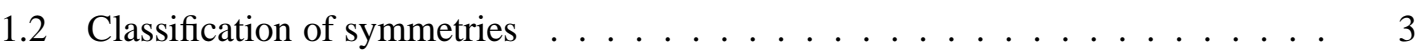

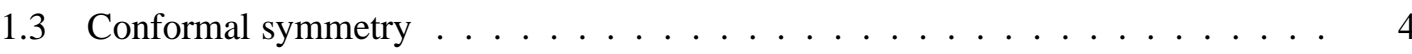

1.4 Curvatures versus potentials $\ldots \ldots \ldots \ldots \ldots \ldots$

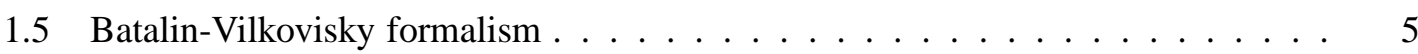

1.6 Local BRST cohomology . . . . . . . . . . . . . . . . . . . 5

1.7 Constraints for variational symmetries . . . . . . . . . . . . . . 6

1.8 Linear Lagrangian theories $\ldots \ldots \ldots \ldots \ldots \ldots$

1.9 Constraints on equations of motion symmetries . . . . . . . . . . 10

1.10 Linear equations of motion . . . . . . . . . . . . . . 10

1.11 Relation to the unfolded formalism . . . . . . . . . . . . . . 11

1.12 Explicit construction of curvature formulations ............. 12

2 Gauge fields in Minkowski spacetime 13

2.1 BRST formulation of Fronsdal fields . . . . . . . . . . . . . 13

2.2 Classification of variational symmetries of a massless scalar . . . . . . . . 16

2.3 Poincaré and dilatation symmetries of Fronsdal fields . . . . . . . . . . 17

2.4 Obstructions to special conformal symmetries for Fronsdal fields . . . . . . . . 18

2.4.1 Obstructions at the level of the action . . . . . . . . . . . . . 18

2.4.2 Obstructions at the level of equations of motion . . . . . . . . 19

2.4.3 Direct obstructions to special conformal generators . . . . . . . . . 21

2.5 Generic massless bosonic fields in Minkowski spacetime . . . . . . . . . . 22

2.5.1 Obstructions at the level of the action . . . . . . . . . . . . 23

2.5.2 Obstructions at the level of equations of motion .......... 24

3 Gauge fields in anti-de Sitter spacetime 24

3.1 Maximal-depth partially-massless fields in $4 \mathrm{~d} \ldots \ldots \ldots . \ldots 24$

3.2 Generic partially-massless gauge fields in AdS . . . . . . . . . . . . . . 27

3.3 Branching rules for modules of the orthogonal algebras . . . . . . . . . 28

3.4 Explicit spin 2 examples . . . . . . . . . . . . . . . . . . . . 29

3.4.1 Maximal-depth partially-massless spin-2 field in 4d . . . . . . . . . 29

3.4.2 Maximal-depth Fradkin-Tseytlin spin-2 field in $4 d$. . . . . . . . . . 31

4 Conclusion 32

Acknowledgments 33 


\section{Generalities}

\subsection{Plan of the paper}

In the first section, we briefly review relevant aspects of symmetries in the context of gauge systems: variational versus equations of motion symmetries, field-theoretic formulation of conformal symmetry, curvature versus potential formulations, the BRST-BV implementation, and the relation to the unfolded approach. We demonstrate that for a gauge system invariant under a global symmetry algebra, the space of global reducibility parameters, and more generally, certain BRST cohomology groups, are necessarily a module thereof. This gives a powerful criterion to analyze whether a given gauge system admits a given global symmetry algebra.

In section 2 we apply this criterion to generic gauge fields in Minkowski space. More precisely, we address the question which general mixed-symmetry bosonic gauge fields on Minkowski space admit an extension from Poincaré to conformal symmetry. We also illustrate the difference between variational and equations of motion symmetries using the simplest example of a massless scalar.

Section 3 is devoted to identifying those gauge fields on anti-de Sitter (AdS) space whose AdS symmetry extends to conformal symmetry. We pay particular attention to the special case of maximal-depth partially-massless (PM) fields in $A d S_{4}$ because these fields have attracted some attention in the literature and can easily be confused with their conformal cousins belonging to the family of (generalized) Fradkin-Tseytlin fields, which we also discuss. We show that these fields are never conformal for $s>1$ neither as gauge fields nor at the level of gauge invariant curvatures. As an illustration the case of $s=2$ is considered in detail.

\subsection{Classification of symmetries}

Algebraic approaches to classifying symmetries of systems of partial differential equations in the context of jet-bundles and the variational bicomplex are by now very welldeveloped, see e.g. [1-4] and also [5-10] for reviews. In particular for Lagrangian systems, symmetries of the action, also called variational symmetries, are a subalgebra of the symmetries of the equations of motion. In applications to fundamental systems, they are privileged since Noether's theorem provides one with a clear procedure on how to implement them in the quantum theory.

The case of Lagrangian gauge systems and of degenerate partial differential equations is less studied in the mathematical literature, mainly because gauge invariance violates technical assumptions needed to apply some of the systematic techniques (see however [11] and references therein). 
For example, for massless higher-spin fields in four-dimensional flat spacetime, symmetries and conservation laws of the equations of motions have been classified in terms of curvatures [12,13] (see also [14] for considerations in higher dimensions), generalizing the result for a massless scalar field [15]. With quantization in mind (see e.g. [16] for an early discussion), suitable potentials and auxiliary fields are introduced in order to make the system Lagrangian, at the expense of introducing gauge symmetries in the massless case [17, 18]. A classification of variational symmetries, and thus also of conservation laws, in such formulations, would be very useful. In particular, one needs to consider suitable equivalence classes of symmetries modulo gauge ones.

\subsection{Conformal symmetry}

Short of a complete classification of symmetries, a standard question is whether a given system admits certain subalgebras of symmetries. Typically, in the situation that we consider below, the relevant systems are by construction invariant under a certain subalgebra of symmetries and one would like to know whether they admit an extension to a bigger algebra of symmetries containing the starting point algebra as a subalgebra. For a variety of field-theoretical realizations of the Poincare or the (anti-) de Sitter algebra for instance, the role of the bigger algebra is played by the conformal algebra.

This question has been thoroughly studied in two related - but in general not entirely equivalent - approaches. The first one is purely representation-theoretical and studies which (A)dS or Poincaré irreps (usually unitary ones) can be lifted to irreps of the conformal group [19-23]. By construction, these considerations concern the gauge invariant spectrum of the theory. The second one is based on equations of motion symmetries, i.e. on (quasi-)invariant differential operators [24, 25, 15, 26]. In particular, a technique to classify linear partial differential equations for which Poincaré lifts to conformal symmetry was developed in [27].

Our considerations in this context will be restricted to free classical (gauge) fields, i.e., to linear PDEs. So we will not address any of the issues raised by the contemporary debate on scale versus conformal invariance for interacting quantum field theories, see e.g. [28, 29] and references therein.

\subsection{Curvatures versus potentials}

Strictly speaking, the symmetry analysis described above applies to PDEs without gauge symmetries. This is often sufficient because the equations of motion of any linear gauge system admit a "curvature" formulation. A standard example consists of Fronsdal fields in (A)dS or Minkowski spacetime which can be reformulated in terms of gauge-invariant curvatures [30]. In the case of spin 1, this is simply the formulation where the Faraday 
tensor $F_{\mu \nu}$ is the fundamental field. For spin 2 (and higher), this is the formulation in terms of the (generalized) Weyl tensor.

It is important to note that the formulation in terms of potentials with gauge symmetries and the associated curvature formulation are not equivalent when insisting on locality. In particular, they may have different symmetries: for instance, at the level of equations of motions, Fronsdal fields in $d=4$ with $s \geqslant 2$ are conformal in terms of curvatures but not in terms of potentials. This is known to experts but we are not aware of a detailed discussion in the literature. In our approach, this is included by using the field-theoretic Batalin-Vilkoviski (BV) formalism, respectively the first quantized BRST approach as described in the next sections, which allows us to provide a simple proof in Section 2.4 below.

\subsection{Batalin-Vilkovisky formalism}

A better technical control on the degeneracies in Lagrangian gauge systems has been achieved with the work of Batalin and Vilkovisky [31-34] (see e.g. [35, 36] for reviews and [37-39] for discussions in the context of jet-bundles).

Let us denote by $\varphi^{i}$ the fields of the theory, by $x^{\mu}$ the spacetime coordinates and by $\mathcal{L}_{0}$ the Lagrangian. Under standard regularity conditions, the notion of a generating set of gauge generators $R_{\alpha}^{i}$ is crucial. Associated to a choice of such a generating set, there is an extended set $\left\{\phi^{A}\right\}=\left\{\varphi^{i}, C^{\alpha}, \cdots\right\}$ of fields $\varphi^{i}$, ghosts $C^{\alpha}$, ghosts for ghosts, ... and their antifields $\phi_{A}^{*}$, graded in terms of a ghost number and equipped with an antibracket

$$
(\cdot, \cdot)=\int d^{n} x \frac{\delta^{R} \cdot}{\delta \phi^{A}(x)} \frac{\delta^{L} \cdot}{\delta \phi_{A}^{*}(x)}-\left(\phi \leftrightarrow \phi^{*}\right)
$$

Furthermore, one can systematically construct a proper, minimal, ghost-number 0 solution

$$
S=\int d^{n} x\left(\mathcal{L}_{0}+\varphi_{i}^{*} R_{\alpha}^{i}\left(C^{\alpha}\right)+\ldots\right)
$$

to the Batalin-Vilkoviski master equation

$$
\frac{1}{2}(S, S)=0
$$

\subsection{Local BRST cohomology}

Once the theory is reformulated within the BV formalism, a natural question is the computation of local BRST cohomology, i.e., the classification of the cohomology of the BRST differential $s=(\cdot, S)$ in the space of local functionals. These groups do not depend on the specific formulation of the theory, in the sense that they can be shown to be invariant under the introduction/elimination of (generalised) auxiliary fields [40]. In particular: 
Equivalence classes of variational symmetries, up to on-shell vanishing variational symmetries and non trivial gauge symmetries with field dependent gauge parameters, are isomorphic to local BRST cohomology in ghost number -1 .

Whereas the computation of $H^{-1}(s)$ is in general rather involved, the computation in lower ghost numbers is much easier. For instance, in irreducible gauge systems for which the generating set of gauge symmetries does not admit local degeneracies, one can show that there is no cohomology in ghost numbers below -2 , while cohomology in ghost number -2 is given by equivalence classes of global reducibility parameters, i.e., by sets of local functions $\bar{f}^{\alpha}$ such that

$$
R_{\alpha}^{i}\left(\bar{f}^{\alpha}\right) \approx 0
$$

where $\approx$ means an equality on the surface defined by the equations and their derivatives, with two sets of local functions considered equivalent if they agree on this surface.

\subsection{Constraints for variational symmetries}

The antibracket induces a well-defined bracket in local BRST cohomology,

$$
(\cdot, \cdot)_{M}: H^{g_{1}}(s) \times H^{g_{2}}(s) \mapsto H^{g_{1}+g_{2}+1}(s)
$$

When $g_{1}=-1=g_{2}$, it follows that $H^{-1}(s)$ is a (graded) Lie algebra with respect to the above antibracket which is isomorphic, up to a change of grading, to the Lie algebra of equivalence classes of variational symmetries. Cohomology in fixed ghost number $H^{g}(s)$ is a module thereof. In turn, this imposes constraints on variational symmetries which we will use in our analysis below. More precisely:

Proposition 1. In the Lagrangian case, local BRST cohomology in the ghost number $g$, $H^{g}(s)$, is necessarily a module of any subalgebra of $H^{-1}(s)$, and thus of any subalgebra of the algebra of equivalence classes of variational symmetries.

Such a property can of course also be established without using the BV formalism. For instance, that global reducibility parameters form a module under variational symmetries has been shown directly in section 3.9 of [41]. The reasoning can be summarized as follows. In proper Lagrangian gauge systems, gauge symmetries form an ideal in the set of all variational symmetries. This implies that, on-shell, the commutator of a gauge symmetry with a global symmetry $\delta_{X}$ can be written in terms of the generating set,

$$
\delta_{X} R_{\alpha}^{i}\left(f^{\alpha}\right)-\delta_{R(f)} X^{i} \approx R_{\alpha}^{i}\left(X_{\beta}^{\alpha}\left(f^{\beta}\right)+\delta_{X} f^{\alpha}\right)
$$

for some total differential operators $X_{\beta}^{\alpha}$. When $f^{\alpha}$ are reducibility parameters, both terms in the commutator on the left hand side vanish on-shell. It follows that $X_{\beta}^{\alpha}+\delta_{X} f^{\alpha}$ are also reducibility parameters. One then proceeds to show that trivial variational symmetries or 
trivial reducibility parameters are mapped to trivial reducibility parameters. This implies that the module action is given by

$$
\left(\left[X^{i}\right],[\bar{f}]\right) \mapsto\left[X_{\beta}^{\alpha}\left(\bar{f}^{\beta}\right)+\delta_{X} \bar{f}^{\alpha}\right] .
$$

In linear theories the generating set of gauge transformations can usually be chosen to be field independent. If the same goes for the reducibility parameters, like in the concrete example of Fronsdal higher-spin fields in dimensions greater than 3 considered below, the equation that determines the module action simplifies to

$$
-\delta_{R(f)} X^{i} \approx R_{\alpha}^{i}\left(X_{\beta}^{\alpha}\left(f^{\beta}\right)\right)
$$

In the case where the linear gauge theory is the result of the linearization of an interacting gauge theory around a solution $\bar{\phi}$, the linear part of gauge transformations with gauge parameters replaced by reducibility parameters, $X^{i}=R_{\alpha}^{i 1}\left(\bar{f}^{\alpha}\right)$, form a subalgebra of variational symmetries. The module action is then described by a derived bracket determined through the terms of the BRST extended cubic vertex of the full interacting theory that contains the information on the gauge algebra, $C_{\alpha}^{*} C_{\beta \gamma}^{\alpha}[\bar{\phi}]\left(C^{\beta}, C^{\gamma}\right)$. This is discussed in detail in sections 4 and 7.4 of [41]. More generally, the derived bracket in the BV formalism has been originally proposed in [42-44].

\subsection{Linear Lagrangian theories}

When the BRST differential $s$ is linear in the fields $\Psi^{\alpha}=\left(\phi^{A}, \phi_{A}^{*}\right)$ of the BV formalism, it is determined by a real "first quantized BRST operator" $\Omega$ (see [45] and also [46] for conventions). Introducing an auxiliary superspace of wave functions $\phi^{\alpha}(x)$ and basis elements $e_{\alpha}$ of opposite ghost number and identical parity to $\Psi^{\alpha}$, and the string field $\Psi=e_{\alpha} \Psi^{\alpha}$, the BRST operator is defined as

$$
s(\Psi)=\Omega(x, \partial) \Psi, \quad \Omega e_{\alpha} \phi^{\alpha}=e_{\beta} \Omega_{\alpha}^{\beta}\left(x, \frac{\partial}{\partial x}\right) \phi^{\alpha}(x),
$$

where $\partial_{\mu}=\frac{\partial}{\partial x^{\mu}}+\Psi_{\mu}^{\alpha} \frac{\partial}{\partial \Psi^{\alpha}}+\ldots$ denotes the total derivative. More generally, any linear differential operator of the form $A_{\beta}^{\alpha}\left(x, \frac{\partial}{\partial x}\right)$ acting from the left determines a unique linear evolutionary vector field acting from the right and such that

$$
V_{A}\left(\Psi^{\alpha}\right)=A_{\beta}^{\alpha}(x, \partial) \Psi^{\beta}
$$

This map is one to one and moreover is a homomorphism, i.e., $\left[V_{A}, V_{B}\right]=V_{[A, B]}$.

In the variational case the space of fields is equipped with a constant nondegenerate odd Poisson bivector $\omega^{\alpha \beta}$. Its inverse $\omega_{\alpha \gamma} \omega^{\gamma \beta}=\delta_{\alpha}^{\beta}$ satisfies $\omega_{\alpha \beta}=(-1)^{1+|\alpha||\beta|} \omega_{\beta \alpha}$ and determines an anti-symplectic form

$$
\omega(\phi, \chi)=\int d^{d} x \omega_{\alpha \beta} \phi^{\alpha}(x) \chi^{\beta}(x), \quad \omega(\phi, \chi)=-(-)^{|\phi||\chi|} \omega(\chi, \phi) .
$$


An operator $A$ is called symplectic if

$$
\omega(A \phi, \chi)+(-)^{|A||\phi|} \omega(\phi, A \chi)=0,
$$

where it is assumed that total derivatives do not contribute because wave functions are assumed to vanish at infinity.

Symplectic operators are one to one with quadratic functionals,

$$
F_{A}=\frac{1}{2} \int d^{d} x \Psi^{\alpha} \omega_{\alpha \beta} A_{\gamma}^{\beta} \Psi^{\gamma} .
$$

The linear vector field $V_{A}$ associated to a symplectic operator $A$ is Hamiltonian, $V_{A}\left(\Psi^{\alpha}\right)=$ $\left(\Psi^{\alpha}, F_{A}\right)$, and satisfies

$$
\left(F_{A}, F_{B}\right)=F_{[A, B]} .
$$

In particular, for linear theories, $\Omega$ is symplectic and the master action can be written as

$$
S=F_{\Omega}, \quad s=(\cdot, S) .
$$

Linear variational symmetries are determined by ghost number -1 quadratic functionals $\mathcal{K}$ such that $(S, \mathcal{K})=0$. They are trivial if $\mathcal{K}=(S, \mathcal{T})$ with $\mathcal{T}$ a quadratic ghost number -2 functional. According to the above discussion, $\mathcal{K}$ is determined by an even ghost number 0 symplectic operator $K$, while $\mathcal{T}$ is odd ghost number -1 symplectic operator $T$, and

$$
[\Omega, K]=0, \quad K \sim K+[\Omega, T] .
$$

The problem of determining linear variational symmetries has thus been rephrased as a problem of BRST operator cohomology. Furthermore, since the Lie algebra structure of equivalence classes of linear variational symmetries is encoded in the antibracket induced in local BRST cohomology quadratic ghost number -1 functionals, it is also represented by the commutator bracket induced in BRST operator cohomology. It follows that:

For linear proper gauge systems, there is thus an isomorphism between the Lie algebras of equivalence classes of linear variational symmetries and the commutator bracket of BRST operator cohomology in the space of symplectic ghost number 0 operators.

As a consequence, we have:

Proposition 2. BRST operator cohomology in the space of ghost number $g$ symplectic operators $H_{\mathrm{sym}}^{g}([\Omega, \cdot])$, is necessarily a module of any subalgebra of $H_{\mathrm{sym}}^{0}([\Omega, \cdot])$, and thus of any subalgebra of the Lie algebra of equivalence classes of linear variational symmetries.

Suppose that one can prove that in a given ghost number $-g$, representatives for local BRST cohomoloy can be chosen to be linear functionals in the fields. This is for instance 
the case in ghost number -1 for the Fronsdal fields discussed below. Without loss of generality, they can always be chosen of the form

$$
L_{\phi}=\int d^{d} x \Psi^{\alpha} \omega_{\alpha \beta} \phi^{\beta}(x)
$$

It then follows from (1.14) that

$$
s\left(L_{\phi}\right) \equiv\left(L_{\phi}, S\right)=(-)^{1+|\phi|} L_{\chi} \quad \Longleftrightarrow \quad \Omega \phi=\chi .
$$

In particular, linear local BRST cohomology in ghost number $g-1$ is isomorphic to BRST state cohomology in ghost number $g, H^{g}(\Omega)$, with vectors of the form $\phi=\phi^{\alpha} e_{\alpha}$.

Independently of this correspondence, we have:

Proposition 3. BRST state cohomology in ghost number $g, H^{g}(\Omega)$, is necessarily a module of any sub-algebra of BRST cohomology for symplectic, ghost number zero operators, $H_{\mathrm{sym}}^{0}([\Omega, \cdot])$, and thus of any subalgebra of the Lie algebra of equivalence classes of linear variational symmetries.

Before making contact with a genuine first-quantized description, note that in this work, the wave functions $\phi^{\alpha}$ and the associated fields $\Psi^{\alpha}$ are taken real from the outset. By using the parity automorphism $I_{\beta}^{\alpha}=(-1)^{|\alpha|} \delta_{\beta}^{\alpha}$, the antisymplectic structure $\omega_{\alpha \beta}$ determines an odd symmetric inner product,

$$
\langle\phi, \chi\rangle=\int d^{d} x(-1)^{|\alpha|} \omega_{\alpha \beta} \phi^{\alpha}(x) \chi^{\beta}(x), \quad\langle\phi, \chi\rangle=(-1)^{|\phi||\chi|}\langle\chi, \phi\rangle .
$$

It turns out that Grassmann odd symplectic operators are formally self-adjoint with respect to (1.19) while Grassmann even symplectic operators are anti-self-adjoint. In particular, $\Omega$ is self-adjoint while representatives of global symmetries are anti-self-adjoint. In concrete application it is often useful to work in term of the symmetric inner product (1.19) in which case the master action takes the form

$$
S=\frac{1}{2}\langle\Psi,-I \Omega \Psi\rangle
$$

Note that $-I \Omega$ is also symplectic and self-adjoint. It is equivalent to $\Omega$ by a change of basis. When working with 1.19 , we implicitly replace in what follows $-I \Omega$ with $\Omega$ and similarly for the representatives of global symmetries, so that the expression for the master action simply becomes $\frac{1}{2}\langle\Psi, \Omega \Psi\rangle$. This does not lead to confusions since $H \cdot(\Omega) \cong H \cdot(-I \Omega)$.

In a full quantum mechanical setting, one deals with a complex Hilbert space and uses a complex structure such that $\omega_{\alpha \beta}$ becomes the imaginary part of the hermitean inner product. This type of construction has been originally used in the context of string field theory, [47-50] (see also [51] for an analysis from the point of view of gauge systems). 


\subsection{Constraints on equations of motion symmetries}

In the non-Lagrangian case, following [45, 46, 52], we assume that the gauge system is described by a nilpotent, ghost number 1 BRST differential $s$ represented by an evolutionary vector field on a bigraded jet-space of fields, which contains, besides the ghost number, an antifield number 11 according to which the BRST differential decomposes as $s=\delta+\gamma+s_{1} \ldots$, with $\delta, \gamma, s_{1}, \ldots$ of antifield number $-1,0,1, \ldots$, such that the cohomology $\delta$ provides a homological resolution of the local functions defined on the surface determined by the original equations of motion. The differential $\gamma$ encodes the gauge symmetries of the equations of motion, which are required to close only on-shell, see e.g. [53, 35] for more details.

When equipped with the commutator bracket, evolutionary vector fields form a graded Lie algebra, the bracket carrying degree 0. Including the BRST differential yields a differential graded Lie algebra, with the bracket descending to cohomology,

$$
[\cdot, \cdot]_{M}: H^{g_{1}}([s, \cdot]) \times H^{g_{2}}([s, \cdot]) \longrightarrow H^{g_{1}+g_{2}}([s, \cdot])
$$

In this context:

Equivalence classes of equations of motion symmetries modulo on-shell vanishing ones and non trivial gauge symmetries are described by the adjoint cohomology of $s$ in the space of evolutionary vector fields of degree $0, H^{0}([s, \cdot])$.

Even though less restrictive than in the Lagrangian case, what we will use to constrain equivalence classes of equations of motion symmetries is:

Proposition 4. The adjoint BRST cohomology $H^{g}([s, \cdot])$ is necessarily a module of any subalgebra of $H^{0}([s, \cdot])$, and thus of any subalgebra of the space of equivalence classes of equations of motion symmetries.

Note that, in addition to equations of motion and Lagrangian systems, one can consider a class of theories interpolating between these two in the sense that the equations of motion are supplemented with a Lagrange structure [54-56].

\subsection{Linear equations of motion}

In the non-Lagrangian case, linear gauge systems are described by a BRST operator $\Omega$ that is no longer required to be symplectic. The adjoint cohomology of $s$ in the space of evolutionary vector fields that are linear in the fields and are of ghost number $g$ is isomorphic to $H^{g}([\Omega, \cdot])$, the adjoint BRST operator cohomology in the space of operators

\footnotetext{
${ }^{1}$ In fact the antifield number is determined by the ghost number and hence is not an independent ingredient of the definition.
} 
of ghost number $g$. Hence, equivalence classes of linear equations of motions symmetries are described by adjoint BRST operator cohomology in degree 0 . The analogs of Propositions 2 and 3 are then:

Proposition 5. The adjoint BRST operator cohomology in ghost number $g, H^{g}([\Omega, \cdot])$, is necessarily a module of any subalgebra of $H^{0}([\Omega, \cdot])$, and thus of any subalgebra of the space of equivalence classes of linear equations of motion symmetries.

Proposition 6. The BRST state cohomology in ghost number $g, H^{g}(\Omega)$, is necessarily a module of any subalgebra of $H^{0}([\Omega, \cdot])$, and thus of any subalgebra of the space of equivalence classes of linear equations of motion symmetries.

Note that, compared to the general case, inequivalent symmetries of linear systems possess a richer structure. Namely, they form an associative algebra with the product induced by the operator product of cohomology representatives.

\subsection{Relation to the unfolded formalism}

Suitable modules under a spacetime symmetry algebra, closely related to BRST state cohomology $H^{g}(\Omega)$, play a crucial role in the unfolded formulation of gauge field dynamics developed in the context of higher-spin theories [57-62]. Typically, the module $H^{-1}(\Omega)$, or $H^{-p}(\Omega)$ with maximal $p$ in general, is an initial ingredient in terms of 1 -form fields. The next step consists in finding modules of 0 -form fields related to gauge invariant curvatures such that the system of 1 and 0 forms is consistent and gauge invariant.

The precise relation to the BRST first quantized formulation can be understood by using the parent approach developed in $[45,63,46,64,65]$. Starting from a free gauge system described by a nilpotent BRST operator $\Omega$ as described in Sections $\mathbf{1 . 8}$ and 1.10 the system is extended by allowing the wave functions to depend on extra variables $y^{\mu}$, which are coordinates on the fibers of the tangent bundle over spacetime, and Grassmann odd ghost variables $\theta^{\mu}, \operatorname{gh}\left(\theta^{\mu}\right)=1$, to be identified with $d x^{\mu}$ and associated to the constraints $\left(\frac{\partial}{\partial x^{\mu}}-\frac{\partial}{\partial y^{\mu}}\right) \Phi=0$. The parent BRST operator taking into account these new constraints along with the original ones accounted in $\Omega$ is

$$
\Omega^{P}=\theta^{\mu}\left(\frac{\partial}{\partial x^{\mu}}-\frac{\partial}{\partial y^{\mu}}\right)+\bar{\Omega}, \quad \bar{\Omega}=\left.\Omega\right|_{x^{\mu} \rightarrow x^{\mu}+y^{\mu}, \frac{\partial}{\partial x^{\mu}} \rightarrow \frac{\partial}{\partial y^{\mu}}} .
$$

Note that (1.22) is a minimal version. In general, one can use a generic parametrization of the tangent space and/or incorporate a suitable (nonlinear) flat connection to account for specific symmetries and/or spacetime geometries. Additional details can be found in [63, 66, 14]. Note in particular that the associated field theories are related through elimination of generalized auxiliary fields, provided the functional space for $y^{\mu}$ is taken to 
be formal power series. This is not the only interesting choice but the one that guarantees equivalence in the sense of local field theories.

Consider the cohomology groups $\mathcal{H}_{p}=H^{-p}\left(\left.\bar{\Omega}\right|_{x}\right)$ of the second term in $\Omega^{P}$ in the space of states at a given spacetime point $x$. It is isomorphic to $H^{-p}(\Omega)$ in the space of formal power series at $x^{\mu}$. In applications, this space is often isomorphic for all spacetime points $x$, as happens for instance if the system has a symmetry group $G$ and is defined on a homogeneous space of $G$. If in addition $\bar{\Omega}$ can be made $x$-independent by a $G$ transformation, i.e., $g \bar{\Omega} g^{-1}$ does not depend on $x$, this transformation makes the first term in (1.22) into a $\mathfrak{g}$-covariant derivative in a specific representation (see [63, 67, 14, 68] for explicit examples and details).

If $\mathcal{H}_{p}$ is $x$-independent, by eliminating generalized auxiliary fields, the system can be reduced to an equivalent system whose states take values in $\mathcal{H}_{p}$ only. More precisely, dynamical fields (in contrast to ghost, antifields, etc.) are $p$-forms $\phi_{p}$ with values in $\mathcal{H}_{p}$. The equations of motion and gauge symmetries for the reduced system then have the following structure

$$
\begin{gathered}
\left(d+\sigma_{1}\right) \phi_{0}=0, \quad\left(d+\sigma_{1}\right) \phi_{1}+\sigma_{2} \phi_{0}=0, \quad\left(d+\sigma_{1}\right) \phi_{2}+\sigma_{2} \phi_{1}+\sigma_{3} \phi_{0}=0, \quad \ldots \\
\delta \phi_{0}=0, \quad \delta \phi_{1}=\left(d+\sigma_{1}\right) \chi_{1}, \quad \delta \phi_{2}=\left(d+\sigma_{1}\right) \chi_{2}+\sigma_{2} \chi_{1}, \quad \ldots
\end{gathered}
$$

where $d=\theta \cdot \frac{\partial}{\partial x}$ and $\sigma_{p}$ are algebraic (i.e., $\frac{\partial}{\partial x}$-independent) operators of order $p$ in $\theta$, and $\chi_{k}$ are gauge parameters which are $k-1$-forms with values in $\mathcal{H}_{k}$. Note that $d+\sigma_{1}+\sigma_{2}+\ldots$ is the homological differential induced by $\Omega^{T}$ in the cohomology of $\bar{\Omega}$. This is the minimal unfolded form of the equations and the BRST state cohomology groups $H^{-p}(\Omega)$ with a suitable choice of the functional space are precisely the spaces of $p$-forms in this formulation.

The cohomology $H^{0}(\bar{\Omega})$ is known in the unfolded approach as the Weyl module. It consists of the gauge invariant (generalized) Weyl tensor together with all of its onshell inequivalent derivatives. This space coincides with the space of gauge-inequivalent solutions to the equations of motions in the space of formal power series.

Let us finally note that $H^{-p}\left(\left.\bar{\Omega}\right|_{x}\right)$ may in general differ in distinct regions of spacetime and then it is not clear what the minimal unfolded formulation is. Typical examples are gauge fields defined on the ambient space $\mathbb{R}^{d+2}$ in the context of the $A d S_{d+1} / C F T_{d}$ correspondence. For instance, in this case $H^{0}\left(\bar{\Omega}_{x}\right)$ for $x$ on the lightcone $X^{2}=0$ and on the hyperboloid $X^{2}=\ell^{2}$ may well be different. It is this fact that underlies the ambient space approach [69,70] to boundary values of $A d S$ gauge fields.

\subsection{Explicit construction of curvature formulations}

Covariant curvature formulations can often be constructed directly from group-theoretical arguments. For instance, such formulations are well-known for 4-dimensional Fronsdal 
fields [71, 72] and Fradkin-Tseytlin fields [73]. For mixed-symmetry massless fields in Minkowski spacetime, they can also be constructed in a direct way [74-76].

In principle, a systematic way to obtain a curvature formulation for a given gauge system uses either the unfolded or the first-quantized BRST approach. Indeed, from (1.11), it follows that $\phi_{0}$ is gauge invariant and that the equation for $\phi_{0}$ does not involve other fields. This means that putting to zero all $\phi_{l}$ with $l>0$ gives a consistent unfolded system. This is the unfolded form of the formulation in terms of curvatures as fundamental fields. The simplest example is Maxwell's equations for the Faraday tensor.

This unfolded formulation of the curvature system is sometimes difficult to construct. In all cases, a simple version of a curvature formulation can be obtained from the parent system (1.22) by putting to zero all fields which are forms of nonzero degree. More precisely, the equations of motion and gauge symmetries then take the form

$$
\left(\frac{\partial}{\partial x^{\mu}}-\frac{\partial}{\partial y^{\mu}}\right) \Phi_{0}(x, y)=0, \quad \bar{\Omega} \Phi_{0}(x, y)=0, \quad \delta_{\chi} \Phi_{0}=\bar{\Omega} \chi,
$$

where $\operatorname{gh}\left(\Phi_{0}\right)=0, \operatorname{gh}(\chi)=-1$ and both $\Phi_{0}$ and $\chi$ are $\theta^{\mu}$-independent. This system is equivalent to the above unfolded formulation if one explicitly eliminates the pure gauge degrees of freedom related to the algebraic gauge symmetries in (1.24). It can thus be regarded as a Stüeckelberg description of the curvature system.

Hence, for general mixed-symmetry (partially)-massless fields in (A)dS or Minkowski space, covariant curvature formulations are implicitly contained in the unfolded or parent formulations constructed in [59,77,-80], respectively [67,68, 81].

\section{Gauge fields in Minkowski spacetime}

\subsection{BRST formulation of Fronsdal fields}

The BRST formulation of higher-spin gauge fields [82-85] can be summarized as follows. Take a $d$-dimensional Minkowski spacetime with $d \geqslant 3$ in order to guarantee regularity assumptions needed below and with metric $\eta_{\mu \nu}=\operatorname{diag}(-1,1 \ldots, 1)$. The space of states is the Fock space of polynomials in bosonic oscillators $a^{\mu}$ (usually denoted by $a_{\mu}^{\dagger}$ ), and fermionic ghosts $b, c$ with $\operatorname{gh}(c)=1, \operatorname{gh}(b)=-1$, tensored with the space of functions in $x^{\mu}$ and the mass-shell ghost $c_{0}, \operatorname{gh}\left(c_{0}\right)=1$. The inner product is $\langle\cdot, \cdot\rangle=\int d^{d} x d c_{0}\langle\cdot, \cdot\rangle_{F}$ where $\langle\cdot, \cdot\rangle_{F}$ is the standard inner product in the Fock space for which $a_{\mu}^{\dagger}=\frac{\partial}{\partial a^{\mu}}, c^{\dagger}=\frac{\partial}{\partial b}$ and $b^{\dagger}=-\frac{\partial}{\partial c}$. The operators $x^{\mu}, c_{0}$ are self-adjoint while ${\frac{\partial}{\partial x^{\mu}}}^{\dagger}=-\frac{\partial}{\partial x^{\mu}}$ and $\frac{\partial}{\partial c_{0}}{ }^{\dagger}=-\frac{\partial}{\partial c_{0}}$.

The self-adjoint BRST operator is

$$
\begin{aligned}
& \Omega=c_{0} \square+c \mathcal{S}+\mathcal{S}^{\dagger} \frac{\partial}{\partial b}+c \frac{\partial}{\partial b} \frac{\partial}{\partial c_{0}}, \quad \Omega^{\dagger}=\Omega, \\
& \square=\frac{\partial}{\partial x} \cdot \frac{\partial}{\partial x}, \quad \mathcal{S}=\frac{\partial}{\partial a} \cdot \frac{\partial}{\partial x}, \quad \mathcal{S}^{\dagger}=-a \cdot \frac{\partial}{\partial x} .
\end{aligned}
$$


The ghost number operator is

$$
\mathcal{G}=c_{0} \frac{\partial}{\partial c_{0}}+c \frac{\partial}{\partial c}-b \frac{\partial}{\partial b}, \quad \mathcal{G}^{\dagger}=1-\mathcal{G} .
$$

Other operators that will be relevant are the BRST invariant extensions of the trace $\mathcal{T}$ and the level $N_{s}$,

$$
\begin{aligned}
& \mathcal{T}=\frac{\partial}{\partial a} \cdot \frac{\partial}{\partial a}+2 \frac{\partial}{\partial b} \frac{\partial}{\partial c} \\
& N_{s}=a \cdot \frac{\partial}{\partial a}+c \frac{\partial}{\partial c}+b \frac{\partial}{\partial b}-s, \quad N_{0}^{\dagger}=N_{0} .
\end{aligned}
$$

The algebra satisfied by these operators is

$$
\begin{aligned}
& {\left[\Omega, N_{s}\right]=[\Omega, \mathcal{T}]=\left[\mathcal{G}, N_{s}\right]=[\mathcal{G}, \mathcal{T}]=0,} \\
& {\left[N_{s}, \mathcal{T}\right]=-2 \mathcal{T}, \quad[\mathcal{G}, \Omega]=\Omega .}
\end{aligned}
$$

The string field is chosen as

$$
\begin{aligned}
\Psi=[ & \Phi\left(x^{\mu}, a^{\mu}\right)+c_{0} b B\left(x^{\mu}, a^{\mu}\right)+c b D\left(x^{\mu}, a^{\mu}\right)+b C\left(x^{\mu}, a^{\mu}\right)+ \\
& \left.-c_{0} \Phi^{*}\left(x^{\mu}, a^{\mu}\right)+c B^{*}\left(x^{\mu}, a^{\mu}\right)+c_{0} c C^{*}\left(x^{\mu}, a^{\mu}\right)+c_{0} c b D^{*}\left(x^{\mu}, a^{\mu}\right)\right]|0\rangle,
\end{aligned}
$$

where the coefficients are expanded as power series in the oscillators $a^{\mu}$. The signs in the expansion have been choosen so that the antibracket between a field and its antifield is 1 . The total ghost number of the string field is 0 and its parity is even. This means that the ghost number and parities of the field coefficients are opposite to those of the states.

We then have

$$
\begin{aligned}
\Omega \Psi=\left[c_{0} \square \Phi\right. & +c \mathcal{S} \Phi+c c_{0} b \mathcal{S} B-c_{0} \mathcal{S}^{\dagger} B+c B+c_{0} c b \square D-c \mathcal{S}^{\dagger} D+ \\
& \left.+c_{0} b \square C+c b \mathcal{S} C+\mathcal{S}^{\dagger} C-c c_{0} \mathcal{S} \Phi^{*}+c_{0} c \square B^{*}+c_{0} c \mathcal{S}^{\dagger} D^{*}\right]|0\rangle .
\end{aligned}
$$

The classical action for a spin $s \geqslant 0$ field is

$$
\begin{aligned}
& S\left[\Psi_{0, s}^{T}\right]=\frac{1}{2}\left\langle\Psi_{0, s}^{T}, \Omega \Psi_{0, s}^{T}\right\rangle, \\
& \mathcal{T} \Psi_{0, s}^{T}=0, \quad N_{s} \Psi_{0, s}^{T}=0, \quad \mathcal{G} \Psi_{0, s}^{T}=0,
\end{aligned}
$$

while the Batalin-Vilkovisky master action is

$$
\begin{aligned}
& S\left[\Psi_{s}^{T}\right]=\frac{1}{2}\left\langle\Psi_{s}^{T}, \Omega \Psi_{s}^{T}\right\rangle, \\
& \mathcal{T} \Psi_{s}^{T}=0, \quad N_{s} \Psi_{s}^{T}=0 .
\end{aligned}
$$

For $d=4$, action (2.7) coincides, up to auxiliary fields, with the gauge theory for free massless fields of helicity $\pm s$ introduced by Fronsdal [17].

Explicitly, by doing the ghost inner product,

$$
\begin{aligned}
S\left[\Psi_{s}^{T}\right]=\frac{1}{2} \int d^{d} x\left[\langle\Phi, \square \Phi\rangle_{F}\right. & -\langle D, \square D\rangle_{F}-2\langle B, \mathcal{S} \Phi\rangle_{F}+2\left\langle B, \mathcal{S}^{\dagger} D\right\rangle_{F}-\langle B, B\rangle_{F} \\
& \left.-2\left\langle\Phi^{*}, \mathcal{S}^{+} C\right\rangle_{F}-2\left\langle D^{*}, \mathcal{S} C\right\rangle_{F}-2\left\langle B^{*}, \square C\right\rangle_{F}\right] .
\end{aligned}
$$


Removing the level $N_{s}$ constraint gives the sum of the free (master) actions for all integer massless spins, while removing the trace constraint $\mathcal{T}$ at fixed spin $s$ gives a model that contains, for $d=4$, massless fields with helicities $-s,-s+2, \ldots, s-2, s$ (see e.g. [86] for a proof in the current context).

Finally, in order to explicitly deal with the trace constraint for Fronsdal fields, we need:

Proposition 7. The Lie algebra of (anti-self-adjoint) operators defined on Ker $\mathcal{T}$ can be described by operators $A$ such that

$$
\mathcal{T} A=B \mathcal{T}, \quad A \sim A+C \mathcal{T}
$$

where $B$ and $C$ are some operators (such that $A$ and $C \mathcal{T}$ are anti-self-adjoint).

The statement is equivalent to the regularity of the equation $\mathcal{T} \phi=0$ or, more precisely, that any operator $C$ such that $C \phi=0$ for all $\phi \in \operatorname{Ker} \mathcal{T}$ can be written as $C=B \mathcal{T}$ for some operator $B$. To see this, note that the Lie algebra spanned by $\mathcal{T}, \mathcal{T}^{\dagger}, N_{1-\frac{d}{2}}$ is isomorphic to $\mathfrak{s l}(2, \mathbb{R})$, which is clear from the identification $E_{+}:=\frac{1}{2} \mathcal{T}^{\dagger}, E_{-}:=-\frac{1}{2} \mathcal{T}$ and $H:=N_{1-\frac{d}{2}}$. Therefore any element in the representation space has a unique decomposition $\Phi=\phi_{0}+\mathcal{T}^{\dagger} \phi_{1}+\left(\mathcal{T}^{\dagger}\right)^{2} \phi_{2}+\ldots$ where $\mathcal{T} \phi_{l}=0$. Moreover, the projector $\Pi$ to the subspace $\operatorname{Ker} \mathcal{T}$ of elements satisfying $\mathcal{T} \chi=0$ can be written as $\Pi=1-\mathcal{T}^{\dagger} \Theta \mathcal{T}$ for some $\Theta\left(H, \mathcal{T}, \mathcal{T}^{\dagger}\right)$. Note that $\operatorname{Ker} \mathcal{T}$ is orthogonal to $\operatorname{Im} \mathcal{T}^{\dagger}$ and $\Pi$ is self-adjoint. Regularity then follows from the structure of the projector $\Pi$. Indeed, $C \phi=0 \forall \phi \in \operatorname{Ker} \mathcal{T}$ implies $C \Pi=0$ which in turn gives $C=C \mathcal{T}^{\dagger} \Theta \mathcal{T}$.

The space of operators on $\operatorname{Ker} \mathcal{T}$ can be identified with the quotient space of operators preserving $\operatorname{Ker} \mathcal{T}$, i.e., $\mathcal{T} A \phi=0 \forall \phi \in \operatorname{Ker} \mathcal{T}$, modulo operators that act trivially, i.e., $A \phi=0 \forall \phi \in \operatorname{Ker} \mathcal{T}$. Thanks to the regularity of $\mathcal{T}$, this space can be written as (2.10) with $B=\mathcal{T} A \mathcal{T}^{\dagger} \Theta$. If one is interested in anti-self-adjoint operators, it is enough to require both $A$ and $C \mathcal{T}$ to be anti-self-adjoint. This completes the proof.

Note that 2.10) is the usual definition of the space of inequivalent linear symmetries of the equation $\mathcal{T} \phi=0$. The above proof applies equally well to the Klein-Gordon equation $\square \varphi=0$ because $\square$ enters an $\operatorname{sl}(2, \mathbb{R})$-algebra together with operators $x^{2}, x \cdot \frac{\partial}{\partial x}+$ $\frac{d}{2}$ so that $(2.10)$ with $\mathcal{T}$ replaced by $\square$ coincides with the definition of linear symmetries for the Klein-Gordon equation [15], discussed here in Section 2.2. Proposition 7, with $\mathcal{T}$ or $\square$, is the first-quantized version of the acyclicity of the associated Koszul differential in the field-theoretical picture.

For our purpose below, it is convenient to characterize operators on $\operatorname{Ker} \mathcal{T}$ differently. Any operator $A$ on the entire representation space determines an operator $\Pi A \Pi$ on $\operatorname{Ker} \mathcal{T}$. Conversely, an operator on $\operatorname{Ker} \mathcal{T}$ can be lifted to the entire space. Using the expression for the projector, one finds that trivial operators on $\operatorname{Ker} \mathcal{T}$, i.e., those satisfying $\Pi A \Pi=0$ 
are of the form $A=\mathcal{T}^{\dagger} \alpha+\beta \mathcal{T}$ for some operators $\alpha, \beta$. It follows that operators on $\operatorname{Ker} \mathcal{T}$ can be described as the quotient space of all operators modulo those of the form $\mathcal{T}^{\dagger} \alpha+\beta \mathcal{T}$. In particular, anti-self-adjoint operators are described by the following quotient

$$
A \sim A+\mathcal{T}^{\dagger} \gamma-\gamma^{\dagger} \mathcal{T}
$$

for some operator $\gamma$. It is important to note that this quotient is only compatible with the commutator, i.e., operators $A^{\prime}=\mathcal{T}^{\dagger} \gamma-\gamma^{\dagger} \mathcal{T}$ equivalent to zero form an ideal in the Lie algebra of anti-self-adjoint operators if one restricts oneselves in addition to operators that preserve $\operatorname{Ker} \mathcal{T}$, i.e. $\mathcal{T} A^{\prime}=\delta \mathcal{T}$ for some $\delta$. Indeed, $\mathcal{T} A^{\prime}=\delta \mathcal{T}$ implies $\mathcal{T} \mathcal{T}^{\dagger} \gamma=$ $\left(\delta-\mathcal{T} \gamma^{\dagger}\right) \mathcal{T}$. Applying $\mathcal{T}^{\dagger} \Theta$ to both sides and using $\Pi \mathcal{T}^{\dagger}=0$ one finds $\mathcal{T}^{\dagger} \gamma=\mathcal{T}^{\dagger} \Theta(\delta-$ $\left.\mathcal{T} \gamma^{\dagger}\right) \mathcal{T}$, so that $A^{\prime}=B \mathcal{T}$ for some $B$. So if we restrict to operators preserving $\operatorname{Ker} \mathcal{T}$ then those of the form $\mathcal{T}^{\dagger} \gamma-\gamma^{\dagger} \mathcal{T}$ form an ideal identical to the one in the proposition 7

\subsection{Classification of variational symmetries of a massless scalar}

Let us now concentrate on a massless scalar, for which $s=0$ in the above description, and use the existing classification of symmetries of the equations of motion [15] to infer the classification of variational symmetries.

In this case, the BRST operator reduces to

$$
\Omega=c_{0} \square,
$$

while the general expression for a ghost number 0 or -1 operators is

$$
\mathbf{A}=A\left(x, \frac{\partial}{\partial x}\right)+B\left(x, \frac{\partial}{\partial x}\right) c_{0} \frac{\partial}{\partial c_{0}}, \quad \mathbf{D}=D\left(x, \frac{\partial}{\partial x}\right) \frac{\partial}{\partial c_{0}} .
$$

The condition that A represents an element of $H^{0}([\Omega, \cdot])$,

$$
\begin{gathered}
{[\Omega, \mathbf{A}]=0, \quad \mathbf{A} \sim \mathbf{A}+[\Omega, \mathbf{D}]} \\
\Longleftrightarrow \quad[\square, A]-B \square=0, \quad A \sim A+D \square, \quad B \sim B+[\square, D],
\end{gathered}
$$

coincides with the definition of linear symmetries used in [15].

The linear space $H^{0}([\Omega, \cdot])$ of inequivalent linear symmetries of the equations of motion (EOM) is an associative algebra $\mathcal{A}$ also known as higher-spin algebra [87]. For a given symmetry $\mathbf{A}$ let $A_{S}(x, p), B_{S}(x, p)$ determine its principal symbols, e.g. $A_{S}$ is the highest derivative term in $A$ where $\frac{\partial}{\partial x^{\mu}}$ is replaced with the commuting variable $p_{\mu}$. It was shown in [15] that (2.14) implies that

$$
p \cdot \frac{\partial}{\partial x} A_{S}(x, p)=p^{2} B_{S}(x, p)
$$

i.e., that $A_{S}$ is a conformal Killing tensor and also that inequivalent linear EOM symmetries are uniquely determined by their principal symbols. It follows that as a linear space, $\mathcal{A}$ is isomorphic to the space of conformal Killing tensors. 
Let us now turn to the space of linear, inequivalent, variational symmetries. According to the general considerations above, they are described by $H^{0}([\Omega, \cdot])$ in the space of (anti)self-adjoint operators. Explicitly,

$$
\mathbf{A}+\mathbf{A}^{\dagger}=0 \Longleftrightarrow A+A^{\dagger}+B=0 .
$$

It is instructive to check that (2.14) and (2.16) imply that $\delta \phi=A \phi$ is indeed a linear variational symmetry associated to

$$
S=\frac{1}{2} \int d^{d} x \phi \square \phi
$$

The elements from $H^{0}([\Omega, \cdot])$ satisfying $\mathbf{A}=-\mathbf{A}^{\dagger}$ form a Lie, but not an associative, subalgebra of $\mathcal{A}$. In this case, (2.16) implies that $A_{S}(x,-p)=-A_{S}(x, p)$ and $B_{S}(x,-p)=B_{S}(x, p)$. In other words:

For a massless real scalar, $H_{\mathrm{sym}}^{0}([\Omega, \cdot])$, the space of inequivalent linear variational symmetries, is isomorphic to the space of conformal Killing tensors of odd rank.

\subsection{Poincaré and dilatation symmetries of Fronsdal fields}

Consider a real spacetime vector field $\xi(x)$ and the anti-self-adjoint, even, ghost number 0 generator

$$
\begin{aligned}
& \Xi=-\left(\xi \cdot \partial+\frac{1}{2} S^{\mu \nu} \Sigma_{\mu \nu}+\frac{\partial \cdot \xi}{d} \Delta+\frac{\partial^{\mu}(\partial \cdot \xi)}{2 d} \kappa_{\mu}\right), \\
& S^{\mu \nu}=\frac{1}{2}\left(\partial^{\nu} \xi^{\mu}-\partial^{\mu} \xi^{\nu}\right), \quad \Sigma_{\mu \nu}=a_{\nu} \frac{\partial}{\partial a^{\mu}}-a_{\mu} \frac{\partial}{\partial a^{\nu}}, \\
& \Delta=\frac{d}{2}-1+2 c_{0} \frac{\partial}{\partial c_{0}}+c \frac{\partial}{\partial c}-b \frac{\partial}{\partial b}, \quad \kappa_{\mu}=4 c_{0}\left(\frac{\partial}{\partial a^{\mu}} b+a_{\mu} \frac{\partial}{\partial c}\right),
\end{aligned}
$$

satisfying

$$
[\mathcal{T}, \Xi]=0=\left[N_{s}, \Xi\right]
$$

By direct computation, one finds

$$
[\Omega, \Xi]=2 \beta^{\lambda}\left(\left(c \frac{\partial}{\partial a^{\lambda}}+a_{\lambda} \frac{\partial}{\partial b}\right) N_{3-\frac{d}{2}}-c a_{\lambda} \mathcal{T}-\mathcal{T}^{\dagger} \frac{\partial}{\partial a^{\lambda}} \frac{\partial}{\partial b}\right) .
$$

if $\xi$ describes infinitesimal conformal transformations,

$$
\partial_{\mu} \xi_{\nu}+\partial_{\nu} \xi_{\mu}=\frac{2}{d} \eta_{\mu \nu} \partial \cdot \xi \Longleftrightarrow \xi_{\mu}=a_{\mu}+\omega_{[\mu \nu]} x^{\nu}+\alpha x_{\mu}+2 x_{\mu} \beta \cdot x-\beta_{\mu} x \cdot x
$$

with constant parameters $a_{\mu}, \omega_{[\mu \nu]}, \alpha, \beta_{\mu}$.

So, the form of the operator $\Xi$ in $(2.18)$ has been fixed by the following requirements: (i) It starts with $-\xi \partial$ implementing the spacetime transformations, (ii) it is antihermitian and (iii) its commutator with the BRST operator produces either zero or, at worst, a 
term which does not depend on the spacetime operators $x^{\mu}, \frac{\partial}{\partial x^{\mu}}$. By themselves, these requirements have lead to conformal vector fields. The conformal transformations and algebra are thus recovered from this construction.

In the current framework, this confirms that infinitesimal Poincaré and dilatation transformations, for which $\beta_{\mu}=0$, are variational symmetries of Fronsdal's higher-spin gauge theory in all dimensions $d \geqslant 3$. Furthermore, for the Klein-Gordon action, for which $s=0$, the same holds for infinitesimal special conformal transformations, since $\left\langle\Psi_{0,0}^{T},[\Omega, \Xi] \Psi_{0,0}^{T}\right\rangle=0$. The explicit form of the generators $P_{\mu}, M_{\mu \nu},-D, K_{\mu}$ are obtained by differentiating $\Xi$ with respect to the parameters.

The last two terms in (2.20) do not contribute for a spin $s$ field because its master action is $S=\frac{1}{2}\left\langle\Psi_{s}^{T}, \Omega \Psi_{s}^{T}\right\rangle$ with $\mathcal{T} \Psi_{s}^{T}=0$ and $N_{s} \Psi_{s}^{T}=0$. Defining $\mathcal{K}=\frac{1}{2}\left\langle\Psi_{s}^{T}, I \Xi \Psi_{s}^{T}\right\rangle$ we thus get,

$$
(S, \mathcal{K})=\frac{1}{2}\left\langle\Psi_{s}^{T},[\Omega, \Xi] \Psi_{s}^{T}\right\rangle=\beta^{\lambda}\left(s-3+\frac{d}{2}\right)\left\langle\Psi_{s}^{T},\left(c \frac{\partial}{\partial a^{\lambda}}+a_{\lambda} \frac{\partial}{\partial b}\right) \Psi_{s}^{T}\right\rangle .
$$

It follows that:

For spin 0, there is conformal invariance at the level of the action in any dimension. For $\operatorname{spin} s=1$, this is the case for $d=4$.

In the next section, we will first use the strategy outlined in Section 1 to quickly show that:

For $d \geqslant 3$, Fronsdal fields with $s \geqslant 2$ are invariant under Poincaré transformations and dilatations, but not conformally invariant, neither at the level of the action, not at the level of the equations of motion.

We will then provide a direct proof that $\Xi$ cannot be modified so as to include special conformal transformations among the variational symmetries, i.e., among the generators commuting with $\Omega$.

\subsection{Obstructions to special conformal symmetries for Fronsdal fields}

\subsubsection{Obstructions at the level of the action}

Local BRST cohomology in ghost number $-2, H^{-2}(s)$, corresponds in the current conventions to BRST state cohomology in ghost number $-1, H^{-1}(\Omega)$, and has been worked out in [88, 45]. This space manifestly enters the unfolded formulation of Fronsdal fields as the module of 1-form fields and has originally appeared in this context in [58]. For a given spin $s$ gauge field, it is represented by the vector space $V$ of elements of the form

$$
b A(x, a)=b a^{\mu_{1}} \ldots a^{\mu_{s-1}} \sum_{m=0}^{s-1} A_{\mu_{1} \ldots \mu_{s-1} \mid \nu_{1} \ldots \nu_{m}} x^{\nu_{1}} \ldots x^{\nu_{m}},
$$


where $A(x, a)$ satisfies

$$
\left(a \cdot \frac{\partial}{\partial x}\right) A=\left(\frac{\partial}{\partial a} \cdot \frac{\partial}{\partial x}\right) A=\left(\frac{\partial}{\partial a} \cdot \frac{\partial}{\partial a}\right) A=\left(\frac{\partial}{\partial x} \cdot \frac{\partial}{\partial x}\right) A=0,
$$

and describes rank $s-1$ traceless Killing tensor fields on Minkowski spacetime. The coefficients $A_{\mu_{1} \ldots \mu_{s-1} \mid \nu_{1} \ldots \nu_{m}}$ are totally traceless and have the symmetries of two-row Young tableaux.

According to Proposition 3 , the vector space $V$ is a module for Poincaré and dilatation transformations, $\Xi V \subset V$ when $\beta_{\mu}=0$ and we will work out the constraints coming from the condition that $V$ be a module under special conformal transformations as well by using standard representation-theoretic arguments.

The subspace $V_{0} \subset V$ annihilated by the translation generators $P_{\nu}=-\frac{\partial}{\partial x^{\nu}}$ is

$$
b a^{\mu_{1}} \ldots a^{\mu_{s-1}} A_{\mu_{1} \ldots \mu_{s-1}},
$$

with symmetric traceless constant tensors $A_{\mu_{1} \ldots \mu_{s-1}}$. The subspace $V_{0}$ is an irreducible $o(d-1,1)$ (i.e. Lorentz) module.

Let us first assume that the action on $V$ of the Poincare algebra extended by dilatations lifts to $o(d, 2)$ by including the special conformal generators $K_{\mu}$. Using the explicit form of the dilatation generator gives $D v=\left(x \cdot \frac{\partial}{\partial x}+\frac{d}{2}-2\right) v$ for $v \in V$. It follows from (2.23) that the spectrum of the dilatation generator is given by $\frac{d}{2}-2, \frac{d}{2}-1, \ldots, \frac{d}{2}-3+s$. At the same time, $D$ can be taken as a generator of an $s l(2)$ subalgebra in $o(d, 2)$, formed by $D, P_{1}, K_{1}$ say. It follows that, in any finite-dimensional module, its spectrum must be symmetric with respect to 0 . This shows that, for $d \geqslant 4$ and $s>0$, the only option is $d=4, s=1$, which is indeed conformal. Formally, in lower dimensions there are extra possibilities: $d=2, s=3$ and $d=3, s=2$. The former does not work because $\operatorname{dim}(V)=2$ and there is no 2-dimensional $\operatorname{sl}(2)$ irreducible representation with weights $-1,0,+1$. The latter is ruled out as all weights of $o(d, 2)$ must be simultaneously either integer or half-integer but $s$ is an integer while the eigenvalues of $D$ are $\pm 1 / 2$.

\subsubsection{Obstructions at the level of equations of motion}

If we are only interested in equations of motion symmetries, the value of the lowest weight $\Delta_{L}$ of the dilatation operator is not known a priori and an extra analysis is needed. Indeed, in the analysis above, this weight was fixed from the requirement that the symmetry generator needed to be anti-self-adjoint.

Let us restrict ourselves to $d \geqslant 3$. Any $o(d, 2)$-module having $V_{0}$ as a Lorentz submodule annihilated by all translation generators $P_{\nu}$, and hence lowest-weight with respect to dilatations, can be induced from $V_{0}$ in a standard way: first pick $\Delta_{L}$, which must be constant on $V_{0}$ because $V_{0}$ is Lorentz irreducible and dilatation generators commute 
with the Lorentz subalgebra, and then consider the (generalized) Verma module generated from $V_{0}$, i.e., consider all formal combinations $K_{\lambda_{1}} \ldots K_{\lambda_{m}} v$ where $v \in V_{0}$. Any $o(d, 2)$ module containing $V_{0}$ as a Lorentz submodule and such that the translation generators $P_{\nu}$ annihilate $V_{0}$ is by construction a quotient of this Verma module, as a consequence of the universality property of Verma modules. Moreover, $\Delta_{L}$ must take special values in order for the quotient to be finite-dimensional. More precisely, $-\Delta_{L}$ has to be integer and such that $-\Delta_{L} \geqslant s-1$. In other words the highest-weight $\left(-\Delta_{L}, s-1\right)$ must be integral dominant. The corresponding finite-dimensional $o(d, 2)$-module is described by a two-row Young tableau (YT) with first row of length $-\Delta_{L}$ and second row of length $s-1$, which will be written $\left(-\Delta_{L}, s-1\right)$. Already for $-\Delta_{L}=s-1$, the spectrum of the dilatation generator contains all integers from $1-s$ to $s-1$ and hence at least $2 s-1$ irreducible Lorentz components. However, the vector space $V$ spanned by elements of the form (2.23) instead contains $s$ irreducible Lorentz components. For $-\Delta_{L}>s-1$, the finite-dimensional $o(d, 2)$-modules with highest-weight $\left(-\Delta_{L}, s-1\right)$ contain even more than $2 s-1$ irreducible Lorentz components. Therefore, the only possibility is the trivial representation: $s-1=\Delta_{L}=0$.

Another way to see that these modules cannot coincide is to observe that the $o(d, 2)$ module associated with the YT $(s-1, s-1)$ is the one of conformal Killing tensor fields of rank $s-1$ in $d$ dimensions. The latter cannot coincide with the Poincare module of usual Killing tensor fields unless it is trivial, i.e., unless $s=1$. In this way, we conclude that Fronsdal fields do not admit special conformal transformations as equations of motion symmetries unless $s=0,1$.

To see that for $s=1$, conformal symmetry is present for $d=4$ only, the argument based on $H^{-1}(\Omega)$ is not enough and $H^{0}(\Omega)$ needs to be analyzed. It is well-known that the space of inequivalent solutions to Maxwell equations is not conformal unless $d=4 \sqrt{2}$. This implies that Fronsdal fields in terms of potentials do not admit conformal symmetry at the level of equations of motion, unless $s=0$ or $s=1, d=4$.

To conclude the discussion of Fronsdal fields in $d=4$, note that, as a linear space, $H^{-1}(\Omega)$ can be made into an $o(4,2)$-module. This does not, however, correspond to an extension of the Poincaré symmetries in the realisation of Subsection $\mathbf{2 . 3}$ and, moreover, it works only for the complexified module because an (anti)self-duality condition should be imposed. The idea is to start with the contragredient module structure on the same linear space $V$ defined in (2.24). For instance, introducing the standard inner product on polynomials, i.e., the one determined by $\langle 1,1\rangle=1, x_{\mu}^{\dagger}=\frac{\partial}{\partial x^{\mu}},\left(a_{\mu}\right)^{\dagger}=\frac{\partial}{\partial a^{\mu}}$ so that for instance $\left\langle x^{\mu}, x^{\nu}\right\rangle=\eta^{\mu \nu}$, and defining new Poincaré generators through $P_{\mu}^{\prime}=-P_{\mu}^{\dagger}$ and $M_{\mu \nu}^{\prime}=-M_{\mu \nu}^{\dagger}$, one finds that the subspace annihilated by $P_{\mu}^{\prime}$ is precisely the Lorentz-

\footnotetext{
${ }^{2}$ See for instance, [27] where conformal equations were classified by listing all suitable conformal modules. In the present language, the cohomology $H^{0}(\Omega)$ is evaluated in the space of formal power series in $x^{\mu}$ in terms of generalized Verma modules.
} 
module associated to the YT $(s-1, s-1)$. Taking $-\Delta_{L}=s-1$ one finds that, as a complex module, $H^{-1}(\Omega)$ lifts to an $o(4,2)$-module described by the YT $(s-1, s-1, s-$ 1). Details of non-branching for this module can be found in Section $\mathbf{3 . 3}$

\subsubsection{Direct obstructions to special conformal generators}

Let us now complete the analysis started in Section 2.3 and show directly that one cannot modify $\Xi$ in (2.18) so as to include special conformal transformations among the variational symmetries when $d \geqslant 3$ and $s \geqslant 2$.

Comparing to equation (II.10) of [89], all the spacetime dependence of the special conformal transformations is correctly reproduced by $\Xi$. It then follows from the analysis in this reference that the only freedom left is to add a spacetime independent operator linear in $\beta_{\lambda}$, or more precisely, to change $\kappa_{\mu}$ to $\widetilde{\kappa}_{\mu}=\kappa_{\mu}+\kappa_{\mu}^{\prime}$ by the addition of a $x^{\mu}, \frac{\partial}{\partial x^{\mu}}$ independent operator $\kappa_{\mu}^{\prime}$ such that

$$
\left[\Delta, \kappa_{\mu}^{\prime}\right]=\kappa_{\mu}^{\prime},\left[\Sigma_{\mu \nu}, \kappa_{\lambda}^{\prime}\right]=\eta_{\mu \lambda} \kappa_{\nu}^{\prime}-\eta_{\nu \lambda} \kappa_{\mu}^{\prime},\left[\kappa_{\mu}, \kappa_{\nu}^{\prime}\right]+\left[\kappa_{\mu}^{\prime}, \kappa_{\nu}\right]+\left[\kappa_{\mu}^{\prime}, \kappa_{\nu}^{\prime}\right]=0
$$

We thus want to show that no such modification allows one to remove the obstruction proportional to $\beta^{\lambda}$ on the right hand side of (2.22).

Using Proposition 7, formulated as in (2.11), a symmetry generator $K$ needs to satisfy $\mathcal{T} K=B \mathcal{T}$ and $[\Omega, K]=\mathcal{T}^{\dagger} \gamma-\gamma^{\dagger} \mathcal{T}$. Combining the ansatz $K=\Xi+\beta^{\mu} \kappa_{\mu}^{\prime}$ with equation (2.20), the no-go result is proven if one can show that there does not exist an operator $\kappa_{\mu}^{\prime}$ independent of $x, \frac{\partial}{\partial x}$ satisfying (2.26) such that

$$
\left[\Omega, \kappa^{\prime \lambda}\right]=-2\left(c \frac{\partial}{\partial a_{\lambda}}+a^{\lambda} \frac{\partial}{\partial b}\right) N_{3-\frac{d}{2}}-A^{\dagger \lambda} \mathcal{T}+\mathcal{T}^{\dagger} A^{\lambda}
$$

for some operators $A^{\lambda}$.

First, using a decomposition according to the degree of homogeneity in $x^{\mu}$, one can take without loss of generality in (2.27) that $\Omega$ reduces to $c \frac{\partial}{\partial b} \frac{\partial}{\partial c_{0}}$ and that $A^{\lambda}$ is $x, \frac{\partial}{\partial x}$ independent.

Second, decomposing operators $A=\sum_{n} A_{n}$ according to the level associated to $N_{0}$, one gets in degree 0 ,

$$
\left[c \frac{\partial}{\partial b} \frac{\partial}{\partial c_{0}}, \kappa_{0}^{\prime \lambda}\right]=-2\left(c \frac{\partial}{\partial a_{\lambda}}+a^{\lambda} \frac{\partial}{\partial b}\right) N_{3-\frac{d}{2}}-A_{2}^{\dagger \lambda} \mathcal{T}+\mathcal{T}^{\dagger} A_{-2}^{\lambda} .
$$

Only this equation is relevant since at level different from zero, the first term on the right hand side does not contribute and one can choose the trivial solution $\kappa_{n}^{\prime \lambda}=0=A_{n-2}^{\lambda}=$ $A_{n+2}^{\dagger \lambda}$.

Third, using the Lorentz transformation properties, one can assume that $\kappa_{0}^{\prime \lambda}=f_{1} \frac{\partial}{\partial a_{\lambda}}+$ $a^{\lambda} g_{-1}$, where $f, g$ depend only on Lorentz invariant combinations of $a, \frac{\partial}{\partial a}$, or, by suitably 
completing these invariants, that $f_{1}=f_{1}\left(\mathcal{T}^{\dagger}, N_{s}, \mathcal{T}, c_{0}, \frac{\partial}{\partial c_{0}}, c, \frac{\partial}{\partial c}, b, \frac{\partial}{\partial b}\right)$ and similarly for $g_{-1}$. Since $c \frac{\partial}{\partial b} \frac{\partial}{\partial c_{0}}$ commutes with $N_{s}$, one can restrict to the zero eigenspace of $N_{s}$, which means in particular that one considers a theory at fixed spin $s$. Writing all operators in normal-ordered form with respect to $\mathcal{T}, \mathcal{T}^{\dagger}$, i.e. in the form $B=\sum_{l, m}\left(\mathcal{T}^{\dagger}\right)^{l} \alpha_{l m}(\mathcal{T})^{m}$ for some $\mathcal{T}, \mathcal{T}^{\dagger}$-independent $\alpha_{l m}$, and using $\left[c \frac{\partial}{\partial b} \frac{\partial}{\partial c_{0}}, \mathcal{T}\right]=\left[c \frac{\partial}{\partial b} \frac{\partial}{\partial c_{0}}, \mathcal{T}^{\dagger}\right]=0$ the lowest order equation gives

$$
\left[c \frac{\partial}{\partial b} \frac{\partial}{\partial c_{0}}, \kappa_{0}^{\prime \lambda}\right]=-2\left(s-3+\frac{d}{2}\right)\left(c \frac{\partial}{\partial a_{\lambda}}+a^{\lambda} \frac{\partial}{\partial b}\right) .
$$

Fourth, decomposing $f_{1}=f_{1}^{0}+c_{0} f_{1}^{1}$, where $f_{1}^{0}$ does not depend on $c_{0}$, and similarly for $g_{-1}$, the equation implies

$$
c \frac{\partial}{\partial b}\left(f_{1}^{1} \frac{\partial}{\partial a_{\lambda}}+a^{\lambda} g_{-1}^{1}\right)=-2\left(s-3+\frac{d}{2}\right)\left(c \frac{\partial}{\partial a_{\lambda}}+a^{\lambda} \frac{\partial}{\partial b}\right) .
$$

Finally, equating $c$-independent terms it follows that $s-3+\frac{d}{2}$ has to vanish, which is only possible for $s=1$ and $d=4$, and for $s=2, d=2$ which is excluded from the discussion.

\subsection{Generic massless bosonic fields in Minkowski spacetime}

Mixed-symmetry massless fields were originally described in [90, 91] while further developments relevant in the present context can be found in [92, 85, 77], and also in [67] which we follow below. These systems are variational and admit a Lagrangian formulation based on a BRST operator $\Omega$ generalizing the first quantized description of Fronsdal fields reviewed in Section $1 \mathbf{1 0}$

In $d$-dimensional Minkowski spacetime, generic mixed-symmetry massless bosonic field of spin $s_{1}, \ldots, s_{p}$, the weights of the respective little group representation, and where the number of rows satisfies $p \leqslant\left[\frac{d-2}{2}\right],[a]$ denotes the integer part of $a \in \mathbb{R}$, can be described by the equations

$$
\begin{gathered}
\frac{\partial}{\partial a_{i}} \cdot \frac{\partial}{\partial a_{j}} \Phi=0, \quad \frac{\partial}{\partial a_{i}} \cdot \frac{\partial}{\partial x} \Phi=0, \quad \frac{\partial}{\partial x} \cdot \frac{\partial}{\partial x} \Phi=0, \\
a_{i} \cdot \frac{\partial}{\partial a_{j}} \Phi=0 \quad i>j, \quad\left(a_{i} \cdot \frac{\partial}{\partial a_{i}}-s_{a}\right) \Phi=0,
\end{gathered}
$$

where we use, as usual, variables $a_{i}^{\mu}$ with $\mu=0, \ldots, d-1$ and $i=1, \ldots, p$ to contract indices and work in terms of a generating function $\Phi$.

In terms of the generating function $\Phi$ the gauge transformations read as

$$
\delta \Phi=Q \chi^{(1)}, \quad Q=\left(a_{i} \cdot \frac{\partial}{\partial x}\right) \frac{\partial}{\partial b_{i}},
$$

where $\chi^{(1)}=b^{i} \chi_{i}^{(1)}(x, a)$. For convenience, we introduced here Grassmann-odd ghost variables $b^{i}$. The same operator $Q$ determines gauge for gauge symmetries $\delta \chi^{(1)}=Q \chi^{(2)}$ 
etc. Gauge (for gauge) parameters satisfy the analog of (2.31) and the following gauge parameter version of (2.32),

$$
\left(a_{i} \frac{\partial}{\partial a_{j}}+b_{i} \frac{\partial}{\partial b_{j}}\right) \chi^{(k)}=s_{i} \delta_{i j} \chi^{(k)} \quad(i \geqslant j), \quad a_{i} \cdot \frac{\partial}{\partial x} \chi^{(k)}=0 .
$$

\subsubsection{Obstructions at the level of the action}

The BRST state cohomology $H^{g}(\Omega)$ for these systems has been computed in [67] and shown to be isomorphic to $H^{g}(Q)$ through the elimination of contractible pairs. It follows that $H^{g}(Q)$ is a module of the global symmetry algebra. It is particularly convenient to consider $H^{-p}(\Omega)$. Recall that $p$ is the number of nonvanishing spin labels $s_{i}$, and hence is the maximal homogeneity degree in $b_{i}$, i.e., the number of rows in the YT describing the field. Indeed, as there are no nonzero elements in degree $<-p$, the coboundary condition is trivial and $H^{-p}(\Omega)$ is given by $\chi^{(p)}=b_{1} \ldots b_{p} \xi(x, p)$ where $\xi$ satisfies

$$
a_{i} \cdot \frac{\partial}{\partial a_{j}} \xi=\delta_{i j}\left(s_{i}-1\right) \xi \quad(i \geqslant j), \quad a_{i} \cdot \frac{\partial}{\partial x} \xi=0 .
$$

along with (2.31).

$H^{-p}(\Omega)$ is a Poincaré-module composed of irreducible Lorentz-modules associated with YT $\left(s_{1}-1, \ldots, s_{p}-1, k\right)$ where $0 \leqslant k \leqslant s_{p}-1$ and $s_{1} \geqslant \ldots \geqslant s_{p}$ [67]. These modules can also be inferred from the unfolded formulation [77]. The subspace $V_{0} \subset H^{-p}(\Omega)$ annihilated by Poincaré translations is an irreducible module with weights $s_{1}-1, \ldots, s_{p}-$ 1.

Repeating the arguments based on the generalized Verma module induced from this $o(d-1,1)$-module one finds that $-\Delta_{L} \geqslant s_{1}-1$ and the decomposition of the corresponding finite-dimensional $o(d, 2)$-module $-\Delta_{L}, s_{1}-1, \ldots, s_{p}-1$ necessarily contains modules not present in the starting point Poincaré-module except if $s_{1}=\ldots=s_{p}=1$ and $\Delta_{L}=0$. The gauge field with such a $H^{-p}(\Omega)$ is a totally-antisymmetric field of rank $p$.

Again, this information infered just from $H^{-p}(\Omega)$ is not enough to conclude for which $p$ a totally antisymmetric field is conformal in $d$-dimensional Minkowski spacetime. Similar to the case of totally symmetric fields, if the system is Lagrangian, the Lagrangian is of second order in derivatives, so that one gets $d / 2-1$ as the weight for the gauge field itself. Furthermore, $H^{-p}(\Omega)$ corresponds to $p$-th level reducibility identities with each level involving first order operators, which gives $\Delta_{L}=\frac{d}{2}-1-p$ for the conformal weight of $V_{0}$. Together with $\Delta_{L}=0$ obtained above, this shows that the only remaining candidates are antisymmetric fields of rank $p=\frac{d}{2}-1$ in (even) dimension $d$, which are indeed known to be conformal. 


\subsubsection{Obstructions at the level of equations of motion}

If one is only interested in EOM symmetries of gauge fields then, in order to see that only rank $\frac{d}{2}-1$ totally antisymmetric gauge fields are conformal, one needs to consider $H^{0}(\Omega)$ as well, i.e., the space of gauge-inequivalent solutions to the EOM. For such fields, this space is a conformal module for $p=\frac{d}{2}-1$ [21,23,27].

As we discussed in 1.12, the analysis of $H^{0}(\Omega)$ is equivalent to an analysis in terms of curvatures because $H^{0}(\Omega)$ is the same for the gauge field and its formulation in terms of curvatures. Let us then briefly review the known results concerning fields in Minkowski spacetime that are conformal in terms of curvatures or, more precisely, which Poincaré irreducible non-gauge fields in Minkowski spacetime are conformal.

It turns out that in odd $d$ only a massless scalar and spinor field are conformal, while in even $d$ there are in addition "spinning" singletons. The latter are fields described by irreducible Lorentz tensors associated to rectangular YT of height $\frac{d}{2}$, which are in particular, traceless and (anti)-selfdual. In fact, they correspond to the massless gauge fields with $p=\frac{d-2}{2}$ and $s_{1}=\ldots=s_{p}=s$, when formulated in terms of curvatures. More precisely, the above irreducible tensors are the gauge-invariant generalized Weyl tensors of these gauge fields. Their conformal invariance was originally shown by identifying those Poincaré irreps that lift to conformal ones [21,23]. In terms of EOM symmetries this follows from the results of [27], while a manifestly local and conformal formulation of these bosonic spinning singletons in terms of curvatures was constructed in [14].

This completes our discussion of possible conformal invariance of bosonic gauge fields on Minkowski spacetime. The extension to fermionic fields is straightforward using e.g. [93, 94]. Note that we have not explicitly discussed massive nor continuous spin representations as they cannot be conformal. This follows essentially from the fact that both of them involve a dimensionful parameter.

\section{Gauge fields in anti-de Sitter spacetime}

\subsection{Maximal-depth partially-massless fields in 4d}

We begin the analysis of possible conformal invariance of $A d S$ gauge fields with the relatively simple, but not so well-known example of totally symmetric partially massless (PM) fields [95-99] of maximal depth $t=s$. In this case the gauge parameter is a scalar. In terms of the $d+1$-dimensional ambient space with coordinates $X^{B},(B=$ $0,1, \cdots, d-1, d)$ and flat metric $\eta_{A B}=\operatorname{diag}(-,+, \cdots,+,-)$, anti-de Sitter spacetime $A d S_{d}$ is the hyperboloid $X \cdot X+1=0$. In these terms, the gauge field is encoded in the 
generating function $\phi(X, A)$ subject to [65, 81, 100, 70]

$$
\begin{gathered}
\left(X \cdot \frac{\partial}{\partial X}+1\right) \Phi=0, \quad X \cdot \frac{\partial}{\partial A} \Phi=0, \quad\left(A \cdot \frac{\partial}{\partial A}-s\right) \Phi=0 \\
\frac{\partial}{\partial X} \cdot \frac{\partial}{\partial X} \Phi=\frac{\partial}{\partial X} \cdot \frac{\partial}{\partial A} \Phi=\frac{\partial}{\partial A} \cdot \frac{\partial}{\partial A} \Phi=0
\end{gathered}
$$

and the gauge transformations

$$
\delta_{\chi} \Phi=\left(A \cdot \frac{\partial}{\partial X}\right)^{s} \chi, \quad\left(X \cdot \frac{\partial}{\partial X}-s+1\right) \chi=0, \quad \frac{\partial}{\partial X} \cdot \frac{\partial}{\partial X} \chi=0 .
$$

The variables $A^{B}, B=0, \ldots, d$ are introduced to contract tensor indices. Note that $\chi$ is $A$-independent.

Just like in the case of Minkowski spacetime fields considered above, it is convenient to introduce a ghost variable $b$ and consider the space of states of the form $\Phi(X, A)+$ $b \chi(X, A)$ with BRST operator $Q=\left(A \cdot \frac{\partial}{\partial X}\right)^{s} \chi \frac{\partial}{\partial b}$ implementing the above gauge equivalence. Although the space of gauge parameters is subject to differential constraints, such a formulation is equivalent to a formulation based on a suitable BRST operator $\Omega$ with free gauge parameters [68]. In particular, $H(\Omega) \cong H(Q)$.

The global reducibility parameters $H^{-1}(\Omega)$ are determined by $\left(A \cdot \frac{\partial}{\partial X}\right)^{s} \chi_{0}=0$. This condition requires $\chi$ to be polynomial in $X$. The first condition in (3.2) fixes the homogeneity of the polynomial to be $s-1$. Finally, the second condition allows one to conclude that $H^{-1}(\Omega)$ is the space of totally traceless rank $s-1$ tensors in $d+1$ dimensions. This is an irreducible module of the $A d S_{d}$ isometry algebra $o(d-1,2)$. Note that irreducibility implies that there can be no gauge symmetries for the gauge parameters in this system.

Following the same idea as before, let us try to check if this $o(d-1,2)$-module can also be an $o(d, 2)$-module. Leaving the rigorous and general proof for the next section, let us present a simple heuristic proof. Observe that all finite-dimensional $o(d, 2)$-modules described by 1-row Young tableaux are simply exhausted by totally traceless fixed rank totally symmetric tensors in $d+2$ dimensions, rather than in $d+1$ dimensions as above. One then concludes that the two spaces do not coincide unless $s=1$. In particular this implies that depth $t=s \mathrm{PM}$ fields in $4 d$ are not conformal as gauge systems, i.e. in terms of potentials, unless $s=1$, in which case it is the usual Maxwell field.

Although maximal-depth PM fields in 4 dimensions are not conformal in general, there exist very similar maximal-depth conformal gauge fields. For $s=1$ they coincide with the Maxwell field, for $s=2$ they were originally found in [96], and for generic $s$ in [101]. They can be seen as higher-depth generalization of usual conformal gauge fields [73], and hence, we call them maximal-depth FT fields below. They belong to the class of conformal gauge fields considered in [62]. Recently they were identified with boundary values of the $A d S_{5}$ maximal-depth PM fields [70]. In $d=4$ these fields have second order equations of motion and gauge transformation of order $s$ in the derivatives. 
More precisely, the flat spacetime Lagrangian for traceless $\varphi_{\mu_{1} \ldots \mu_{s}}(x)$ reads as [101]

$$
L=\partial^{\nu} \varphi^{\mu_{1} \ldots \mu_{s}} \partial_{\nu} \varphi_{\mu_{1} \ldots \mu_{s}}-\frac{2 s}{s+1} \partial_{\nu} \varphi^{\nu \mu_{2} \ldots \mu_{s}} \partial^{\lambda} \varphi_{\lambda \mu_{2} \ldots \mu_{s}}
$$

and is invariant under $\delta \varphi_{\mu_{1} \ldots \mu_{s}}=\partial_{\mu_{1}} \ldots \partial_{\mu_{s}} \chi-$ traces. Thanks to conformal invariance, they can be seen as fields on any conformally flat space and, in particular, on $A d S_{4}$. A natural question is then what their relationship to the maximal-depth PM fields on the same spacetime precisely is.

To answer this question, let us consider again global reducibilities. Using the ambient formulation of [70], the space of reducibilities can be described in terms of polynomials in $d+2$-variables $X^{M}$ satisfying

$$
X^{M} \frac{\partial}{\partial X^{M}} \Phi=(s-1) \Phi, \quad \eta^{M N} \frac{\partial}{\partial X^{M}} \frac{\partial}{\partial X^{N}} \Phi=0 .
$$

This subspace is determined by the same equations as $H^{-1}(\Omega)$ above but in $d+2$ dimensions. Unless $s=1$ these spaces do not coincide. In Section $\mathbf{3 . 4}$ we explicitly compare these two fields in the first nontrivial case of $s=2$.

As far as totally symmetric PM fields of maximal depth are concerned, one can wonder if, similarly to Fronsdal fields in 4 dimensions, the equations of motion are conformal in terms of curvatures. To answer this question we use the formulation in terms of curvatures proposed in [102] (see e.g. Sec. 3.4 for the simplest non trivial example of $s=2$ ). If these systems were conformal, one could equally well rewrite them in flat Minkowski spacetime using a Weyl transformation. As the flat limit for these AdS systems is regular, its Weyl transformation to flat space should coincide with its naive flat limit obtained by putting the cosmological constant to zero. More precisely, for the flat limit of a PM maximal-depth field, the fundamental field is an irreducible Lorentz tensor $F_{\mu_{1} \ldots \mu_{s} \mid \nu}$, i.e., it is symmetric over all $\mu$ indices and such that the complete symmetrization over all lower indices gives zero. It then follows from the classification results of [27] that, for such a Lorentz tensor field labelled by a "hook" YT $(s, 1)$, there are only two conformal equations which are first-order in derivatives and a rank- $s$ Lorentz tensor: one is a totally symmetric rank-s Lorentz tensor with conformal weight 2 while the other one is labelled by a hook YT $(s-1,1)$ and has conformal weight $s+3$. The former equation corresponds to the curvature formulation of a maximal-depth conformal gauge field which differs from the corresponding PM field unless $s=1$. This difference is explicitly illustrated on the example of $s=2$ in Sec. 3.4 below. The latter equation also differs from the corresponding PM field since in particular, the curvature has a different conformal weight. In conclusion: 3

\footnotetext{
${ }^{3}$ At first glance, this conclusion differs from [103] but this paper is based on different assumptions and makes use of a different definition of symmetries. In particular, the $o(4,2)$ symmetry discussed in [103] does not seem to correspond to standard conformal spacetime transformations.
} 
Maximal-depth PM field with $s>1$ are not conformal, neither in terms of potentials, nor in terms of curvatures.

\subsection{Generic partially-massless gauge fields in AdS}

A partially massless bosonic gauge field in $A d S_{d}$ is determined by a finite-dimensional module of $o(d-1)$ with weights (spins) $s_{1}, \ldots, s_{r}$. Here $r=\left[\frac{d-1}{2}\right]$ is the rank of a rotation subalgebra $o(d-1)$, while $s_{1} \geqslant \ldots \geqslant s_{r}, p$ and $t$ are integer parameters, $1 \leqslant p \leqslant r$ and $1 \leqslant t \leqslant s_{p}-s_{p+1}$. This corresponds to a (partially)-massless field of spin $s_{1}, \ldots, s_{r}$ with depth- $t$ gauge transformation associated to the $p$-th row. More details can be found in [104, 59, 78, 68, 81].

The BRST first-quantized description for a generic bosonic gauge field on AdS has been constructed in [68, 81] (see also [63, 59, 78, 105, 79] for earlier related work). The nontrivial $H^{-i}(\Omega)$ are in degree 0 and $p$. For an irreducible (partially)-massless field, the space $H^{-p}(\Omega)$ is a finite-dimensional irreducible $o(d-1,2)$-module with highest weight $s_{1}-1, \ldots, s_{p-1}-1, s_{p}-1, s_{p}-t, s_{p+1}, \ldots, s_{r}$, i.e. the module described by the Young diagram with the lengths of rows given by: 4

$$
s_{1}-1 \geqslant \ldots \geqslant s_{p-1}-1 \geqslant s_{p}-1 \geqslant s_{p}-t \geqslant s_{p+1} \geqslant \ldots \geqslant s_{r} .
$$

Note the row of length $s_{p}-1$ in the middle of the diagram and a subsequent row of length $s_{p}-t$. For instance, for $d=4$ and $t=1$, one gets the familiar 2-row rectangular tableaux of length $s_{1}-1$. Note that $r=1$ in this case.

According to the $o(d+2) \downarrow o(d+1)$ branching rules summarized in the next subsection, if module (3.5) is nontrivial, it can be lifted to $o(d, 2)$ iff $d$ is even and this Young tableau is rectangular of height $\frac{d}{2}$. This condition resricts $s_{i}$ in such a way that $s_{1}-1=s_{2}-1 \ldots=s_{p}-1=s_{p+1}=\ldots=s_{r}$ so that according to [22] the field belongs to the class of unitary mixed-symmetry fields. In particular, $t=1$ so that mixed symmetry PM fields cannot be conformal in general.

To obtain further restrictions one has to consider $H^{0}(\Omega)$ as well. According to the analysis of [22] unitary AdS fields may admit conformal symmetry only for $s_{1}=\ldots=s_{r}$ (in particular $p=r$ ) and $d$ even. If we restrict ourselves to the case $p=r, H^{-p}(\Omega)$ is associated to a spinning singleton [20-22]. As an $o(d, 2)$ module, $H^{-p}(\Omega)$ is a finitedimensional module described by a rectangular tableau of height $\frac{d}{2}+1$ and length $s-$ 1. The module is realized by (anti)-selfdual tensors of this symmetry type in $d+2$ dimensions. In dimensions $d$ different than $2 \bmod 4$ however, modules of this sort are necessarily complex as the (anti-) selfduality condition does not have real solutions in

\footnotetext{
${ }^{4}$ It is this module where a $p$-form field takes values in the unfolded description [59,78] of AdS gauge fields.
} 
such dimensions 5 . In particular, this implies that in $A d S_{d}$ with $d=4,8, \ldots$ real fields can be conformal in terms of potentials only for $s_{1}=\ldots=s_{r}=1$, i.e., when the module is trivial. These are totally antisymmetric fields of maximal rank which are known to be conformal for even $d$.

An interesting question is whether spinning singletons in $A d S_{d}$ with $d \geqslant 6, d=$ $2 \bmod 4$ and $s>1$ can be conformal in terms of potentials. Note that those with $s=1$ are conformal in terms of potentials, while they all are known to be conformal in terms of curvatures. The necessary condition advocated here does not exclude this possibility and resolving the issue requires further study.

\subsection{Branching rules for modules of the orthogonal algebras}

The branching rules of a Lie algebra $\mathfrak{g}$ describe the decomposition of its irreps restricted to a subalgebra $\mathfrak{h}$. We will be interested in the very exceptional case when the $\mathfrak{g}$-irrep remains irreducible under the restriction $\mathfrak{g} \downarrow \mathfrak{h}$, i.e., when the decomposition contains only a single $\mathfrak{h}$-irrep with multiplicity one. The trivial representation is an obvious example of such an irrep. The branching rules of classical algebras are well-known for finite dimensional irreps while the problem is obviously more involved for infinite-dimensional ones.

The importance of branching rules for our purpose is the following fact: An h-irrep can be lifted to a $\mathfrak{g}$-irrep if and only if this $\mathfrak{h}$-irrep is the only irrep appearing in the restriction $\mathfrak{g} \downarrow \mathfrak{h}$ of the $\mathfrak{g}$-irrep. In other words, there is a one-to-one correspondence between the $\mathfrak{h}$-irreps that can be lifted to $\mathfrak{g}$-irreps and the $\mathfrak{g}$-irreps that remains irreducible under the restriction $\mathfrak{g} \downarrow \mathfrak{h}$.

To see which finite-dimensional $o(d-1,2)$-modules can be lifted to $o(d, 2)$, we recall the basic facts on $o(d) \downarrow o(d-1)$ branching rules. The finite-dimensional irreducible $o(d)$-module characterized by the dominant integral $o(d)$-weight $\vec{s} \equiv\left(s_{1}, \ldots, s_{r}\right)$ will be denoted by $\mathcal{D}_{o(d)}(\vec{s})$. Here $r$ denotes the rank of $o(d)$, i.e., the integer part of $d / 2$. The "spin" labels of the weight $r$-vector $\vec{s}$ are either all integers or all half-integers, and they satisfy

$$
\begin{aligned}
& s_{1} \geqslant \ldots \geqslant s_{r} \geqslant 0 \quad \text { for } d=2 r+1 \\
& s_{1} \geqslant \ldots \geqslant s_{r-1} \geqslant\left|s_{r}\right| \text { for } d=2 r .
\end{aligned}
$$

When $d=2 r$, the last label $s_{r}$ can be positive or negative. The integer part of the (absolute values) of the components in $\vec{s}$ define a Young diagram where each spin label gives the length of the corresponding row.

\footnotetext{
${ }^{5}$ This is in agreement with [106] where the conformal invariance of doubled (complexified) sets of totally-symmetric fields in $A d S_{4}$ was put forward.
} 
The classical branching rules for the restriction $o(d) \downarrow o(d-1)$ of finite-dimensional irreducible modules can be expressed as follows:

$$
\mathcal{D}_{o(d)}(\vec{s}) \downarrow \bigoplus_{\vec{t}} \mathcal{D}_{o(d-1)}(\vec{t})
$$

where the direct sum is over all $o(d-1)$-weights $\vec{t}$ such that

$$
\begin{aligned}
& s_{1} \geqslant t_{1} \geqslant \ldots \geqslant s_{r-1} \geqslant t_{r-1} \geqslant s_{r} \geqslant\left|t_{r}\right| \text { for } d=2 r+1, \\
& s_{1} \geqslant t_{1} \geqslant \ldots \geqslant s_{r-1} \geqslant t_{r-1} \geqslant\left|s_{r}\right| \text { for } d=2 r,
\end{aligned}
$$

with entries in $\vec{s}$ and $\vec{t}$ which are simultaneously all integers or all half-integers.

Lemma 8. A nontrivial irreducible $o(d)$-module $\mathcal{D}_{o(d)}(\vec{s})$ remains irreducible after its restriction to $o(d-1)$ if and only if $d=2 r$ and $s_{1}=\ldots=s_{r-1}=\left|s_{r}\right|$, i.e., if it is described by a rectangular Young diagram of height $d / 2$.

Proof. The branching rules (3.8) and (3.9) imply the following chain of inequalities $s_{1} \geqslant$ $t_{1} \geqslant \ldots \geqslant s_{r-1} \geqslant t_{r-1} \geqslant\left|s_{r}\right|$ which are valid in any $d$. One can see that a necessary condition in order to have a single allowed set of components $t_{1}, \ldots, t_{r-1}$ is that $s_{1}=$ $\ldots=s_{r-1}=\left|s_{r}\right|$. For $d=2 r$, this fixes uniquely $\vec{t}$ to be the $(r-1)$-vector (since $o(d-1)$ has rank $r-1$ ) such that $t_{1}=\ldots=t_{r-1}=\left|s_{r}\right|$. For $d=2 r+1$, inspecting the last inequality $s_{r} \geqslant\left|t_{r}\right|$ in the branching rule (3.8), one can see that $s_{r}$ must vanish in order to have a single allowed component $t_{r}$. This implies that the trivial irreducible $o(d)$-module $\mathcal{D}_{o(d)}(\overrightarrow{0})$ is the only one that remains irreducible after restriction to $o(d-1)$ for $d$ odd.

An obvious corollary is that, if one performs two such branchings, the only irreducible $o(d)$-module which remains irreducible after its restriction to $o(d-2)$ is the trivial module.

\subsection{Explicit spin 2 examples}

To illustrate the difference between $s=t=2$ PM field and FT field in 4d, let us work in terms of tangent tensors.

\subsubsection{Maximal-depth partially-massless spin-2 field in 4d}

$s=t=2$ PM field in $d=4$ in terms of potentials: Following [95, 96], the equations of motion for a $s=t=2 \mathrm{PM}$ field in $4 \mathrm{~d}$ are

$$
\begin{aligned}
\left(\nabla^{2}+4 \mu^{2}\right) \varphi_{\mu \nu}-\left(\nabla_{\mu} \nabla^{\rho} \varphi_{\rho \nu}+\nabla_{\nu} \nabla^{\rho} \varphi_{\rho \mu}\right) & +\nabla_{\mu} \nabla_{\nu} \varphi^{\prime}- \\
& -g_{\mu \nu}\left(\left(\nabla^{2}+\mu^{2}\right) \varphi^{\prime}-\nabla^{\rho} \nabla^{\sigma} \varphi_{\rho \sigma}\right)=0,
\end{aligned}
$$


where $\varphi^{\prime} \equiv g^{\mu \nu} \varphi_{\mu \nu}$. Here $\mu=L^{-1}$ the inverse AdS radius so that e.g. $R_{\mu \nu \rho \sigma}=$ $-\mu^{2}\left(g_{\mu \rho} g_{\nu \sigma}-g_{\nu \rho} g_{\mu \sigma}\right)$. The equations are invariant under the following gauge symmetry

$$
\delta_{\xi} \varphi_{\mu \nu}=\left(\nabla_{\mu} \nabla_{\nu}-\mu^{2} g_{\mu \nu}\right) \xi
$$

with unconstrained scalar parameter $\xi(x)$.

Equations (3.10) have differential consequences of first order [97]. Applying $\nabla^{\mu}$ to both sides of (3.10) one finds

$$
\nabla^{\mu} \varphi_{\mu \nu}-\nabla_{\nu} \varphi^{\prime}=0, \quad \varphi^{\prime} \equiv g^{\mu \nu} \varphi_{\mu \nu}
$$

Let us also present the partially gauge fixed version of this system. Namely, let us consider the gauge condition $\varphi^{\prime}=0$. Its variation under a gauge transformation is given by

$$
\delta \varphi^{\prime}=\left(\nabla^{2}-4 \mu^{2}\right) \xi,
$$

so that the gauge is reachable. Indeed, in the context of jet-spaces, any element is in the image of $\nabla^{2}$. The gauge fixed system reads

$$
\begin{aligned}
& \left(\nabla^{2}+4 \mu^{2}\right) \varphi_{\mu \nu}=0, \quad \nabla^{\mu} \varphi_{\mu \nu}=0, \quad g^{\mu \nu} \varphi_{\mu \nu}=0 \\
& \delta \varphi_{\mu \nu}=\left(\nabla_{\mu} \nabla_{\nu}-\mu^{2} g_{\mu \nu}\right) \xi, \quad\left(\nabla^{2}-4 \mu^{2}\right) \xi=0 .
\end{aligned}
$$

This formulation can be rewritten in ambient terms by identifying $\varphi_{\mu \nu}$ with the pullback of ambient $\varphi_{A B}$ satisfying $X^{A} \varphi_{A B}=0,\left(X \cdot \frac{\partial}{\partial X}+1\right) \varphi_{A B}=0$, and similarly for the gauge parameter.

The space of global reducibilities is determined by $\delta_{\xi} \varphi_{\mu \nu}=0$. The consequence $g^{\mu \nu} \delta_{\xi} \varphi_{\mu \nu}=0$ reads explicitly

$$
\left(\nabla^{2}-4 \mu^{2}\right) \xi=0 \text {. }
$$

Let us identify $\xi$ as the pullback of $\Xi(X)$ defined on ambient space $\mathbb{R}^{3+2}$ and satisfying $\left(X \cdot \frac{\partial}{\partial X}-1\right) \Xi=0, \frac{\partial}{\partial X} \cdot \frac{\partial}{\partial X} \Xi=0$. In terms of $\Xi$, the gauge transformation is $\partial_{A} \partial_{B} \Xi$ and hence $\Xi$ must be polynomial. One concludes that $\Xi=\xi_{A} X^{A}$, so that reducibilities are parametrized by $d+1$ dimensional ambient vectors.

$s=t=2$ PM field in $d=4$ in terms of curvatures: Following [102], the curvature is given by

$$
F_{\mu \nu \mid \rho}=\nabla_{\mu} \varphi_{\nu \rho}-\nabla_{\nu} \varphi_{\mu \rho}
$$

In terms of $F_{\mu \nu \mid \rho}$, equations of motion (3.10) take the form

$$
\nabla^{\rho} F_{\rho(\mu \mid \nu)}-g_{\mu \nu} \nabla^{\rho} F_{\rho}^{\prime}+\nabla_{(\mu} F_{\nu)}^{\prime}=0
$$

where $F_{\mu}^{\prime}=F_{\mu \rho \mid \nu} g^{\rho \nu}$ and $X_{(a} Y_{b)}=\frac{1}{2}\left(X_{a} Y_{b}+X_{b} Y_{a}\right)$. In this form, the equations of motion follow from the Lagrangian [102]:

$$
L^{P M}=F_{\mu \nu \mid \rho} F^{\mu \nu \mid \rho}+F^{\prime \nu} F_{\nu}^{\prime} .
$$


If one treats $F_{\mu \nu \mid \rho}$ as the fundamental field, one also needs to add algebraic conditions and Bianchi identities so that the complete set of equations becomes

$$
\begin{gathered}
F_{\mu \nu \mid \rho}=-F_{\nu \mu \mid \rho}, \quad F_{[\mu \nu \mid \rho]}=0, \quad F_{\mu \nu \mid \rho} g^{\nu \rho}=0, \\
\nabla^{\mu} F_{\mu \nu \mid \rho}=0, \quad \nabla_{[\sigma} F_{\mu \nu] \mid \rho}=0 .
\end{gathered}
$$

Note that if $F_{\nu \mu \mid \rho}$ is (anti)-selfdual the last two equations are equivalent.

\subsubsection{Maximal-depth Fradkin-Tseytlin spin-2 field in 4d}

$s=t=2$ FT field in $d=4$ in terms of potentials: Another related system in $4 \mathrm{~d}$ was also proposed in [95,96] (see also references therein). The equations of motion have the form

$$
\left(\nabla^{2}+4 \mu^{2}\right) \varphi_{\mu \nu}-\frac{2}{3}\left(\nabla_{\mu} \nabla^{\rho} \varphi_{\rho \nu}+\nabla_{\nu} \nabla^{\rho} \varphi_{\rho \mu}\right)+\frac{1}{3} g_{\mu \nu} \nabla^{\rho} \nabla^{\sigma} \varphi_{\rho \sigma}=0
$$

and $g^{\mu \nu} \varphi_{\mu \nu}=0$. The gauge law is

$$
\delta_{\xi} \varphi_{\mu \nu}=\left(\nabla_{\mu} \nabla_{\nu}-\frac{1}{4} g_{\mu \nu} \nabla^{2}\right) \xi
$$

with $\xi$ unconstrained. This system is conformal and can be identified [70] with the boundary value of the $t=s=2 \mathrm{PM}$ field on $A d S_{5}$.

In contrast to the $s=t=2 \mathrm{PM}$ field considered above, the gauge $\nabla^{\mu} \varphi_{\mu \nu}=0$ is not reachable in general. On the contrary, $V_{\mu}:=\nabla^{\mu} \varphi_{\mu \nu}$ satisfy Maxwell's equations and transform as $\delta V_{\mu}=\frac{3}{4} \nabla_{\mu}\left(\nabla^{2}-4 \mu^{2}\right) \xi$.

To see what this system describes, let us decompose $\varphi_{\mu \nu}$ (in a nonlocal way) into $\varphi_{\mu \nu}^{0}$ satisfying $\nabla^{\mu} \varphi_{\mu \nu}^{0}=0$ and $V_{\mu}$ describing the rest. The equations for $\varphi_{0}$ reduce to (3.14), so that a FT field with $s=t=2$ decomposes into a PM field $\varphi^{0}$ with $s=t=2$ and a Maxwell field $V$ with $s=t=1$.

The space of global reducibilities is given by solutions to $\left(\nabla_{\mu} \nabla_{\nu}-\frac{1}{4} g_{\mu \nu} \nabla^{2}\right) \xi=0$. Let us consider first the consequence $\nabla^{\mu} \delta_{\xi}\left(\varphi_{\mu \nu}\right)=0$, or explicitly,

$$
\nabla^{\mu} \delta_{\xi}\left(\varphi_{\mu \nu}\right)=\frac{3}{4} \nabla_{\nu}\left(\nabla^{2}-4 \mu^{2}\right) \xi=0
$$

The general solution to this equation has the form $\xi=a+\xi_{0}$ where $a$ is constant and $\xi_{0}$ is a general solution to $\left(\nabla^{2}-4 \mu^{2}\right) \xi_{0}=0$. In turn, just like in the case of a PM field, it is convenient to represent $\xi$ as the pullback to the hyperboloid of $\Xi_{0}$ defined on $\mathbb{R}^{3+2}$ and satisfying $\frac{\partial}{\partial X} \cdot \frac{\partial}{\partial X} \Xi_{0}=0,\left(X \cdot \frac{\partial}{\partial X}-1\right) \Xi_{0}=0$. In terms of the ambient space, conditions $\delta_{\xi_{0}} \varphi_{\mu \nu}=0$ take the form $\partial_{A} \partial_{B} \Xi_{0}=0$ where $\left(\nabla^{2}-4 \mu^{2}\right) \xi_{0}=0$ has been taken into account. So the solution is again given by $\Xi_{0}=\xi_{A} X^{A}$. Putting everything together, the general solution for $\xi$ is $\xi=a+\xi_{A} X^{A}(x)$ and the space of reducibilities is 6 -dimensional, confirming the conclusion of the manifestly conformal considerations of Section 3.1. Let us stress that in contrast to Section 3.1 we now have not assumed that conformal symmetry is realized on gauge parameters. 
$s=t=2$ FT field in $d=4$ in terms of curvatures: The traceless component of the curvature is

$$
\widetilde{F}_{\mu \nu \mid \rho}=\nabla_{\mu} \varphi_{\nu \rho}-\nabla_{\nu} \varphi_{\mu \rho}-\frac{1}{3} g_{\mu \rho} \nabla^{\alpha} \varphi_{\alpha \nu}+\frac{1}{3} g_{\nu \rho} \nabla^{\alpha} \varphi_{\alpha \mu}
$$

In terms of $\widetilde{F}$, the equation of motion take the form

$$
\nabla^{\mu} \widetilde{F}_{\mu(\nu \mid \rho)}=0
$$

They follow from the Lagrangian

$$
L^{F T}=\frac{1}{2} \widetilde{F}_{\mu \nu \mid \rho} \widetilde{F}^{\mu \nu \mid \rho} .
$$

If one treats $\widetilde{F}_{\mu \nu \mid \rho}$ as the fundamental fields, the complete set of equations is

$$
\begin{gathered}
\widetilde{F}_{\mu \nu \mid \rho}=-\widetilde{F}_{\nu \mu \mid \rho}, \quad \widetilde{F}_{[\mu \nu \mid \rho]}=0, \quad \widetilde{F}_{\mu \nu \mid \rho} g^{\nu \rho}=0, \\
\nabla^{\mu} \widetilde{F}_{\mu(\nu \mid \rho)}=0, \quad \nabla_{[\sigma} \widetilde{F}_{\mu \nu] \mid \rho}=g_{\rho[\sigma} A_{\mu \nu]},
\end{gathered}
$$

where $A_{\mu \nu}$ is an antisymmetric tensor. The last equations can be written as $\mathcal{P}\left(\nabla_{[\sigma} \widetilde{F}_{\mu \nu] \mid \rho}\right)=$ 0 , where $\mathcal{P}$ denotes the projector to the totally traceless component. Note that if $\widetilde{F}_{\nu \mu \mid \rho}$ is (anti)-selfdual, the last two equations are equivalent.

By comparing (3.27), (3.28) to (3.19), (3.20), one observes that the $s=t=2$ FT equations of motion are a subset of the $s=t=2 \mathrm{PM}$ equations. Therefore, the space of solutions of the $s=t=2 \mathrm{PM}$ equations is a subspace of the $s=t=2$ FT one. Indeed, the former is an $o(d-1,2)$-submodule of the latter. The crucial point is that, nevertheless, the former is not an $o(d, 2)$-submodule of the latter because the extra equations of the $s=$ $t=2 \mathrm{PM}$ field are not conformally invariant for the conformal weight of the $s=t=2$ FT field. The same remains true for $s>2$.

\section{Conclusion}

In this work we have studied structural properties of global symmetries in gauge systems. In particular, in the context of the BV-BRST approach, we have shown that BRST cohomology in the space of local functionals, $H^{-p-1}(s)$, as well as BRST-state cohomology $H^{-p}(\Omega)$ in the case of linear systems, are necessarily modules over any subalgebra of the algebra of global symmetries.

Of special importance are "global reducibility parameters" which correspond to these cohomology groups for $p \geqslant 1$. In contrast to BRST cohomology groups in other ghost numbers, global reducibilities are typically finite-dimensional. This makes them especially useful in order to constrain global symmetries since the analysis then only requires 
standard tools from representation theory. Surprisingly, in the particular examples where we study which (A)dS or Poincaré gauge fields admit conformal symmetry, this analysis is powerful enough to rule out most of the candidates, without analyzing the space of solutions.

Our approach is closely related to the unfolded formalism. Namely, in the unfolded approach, the construction of gauge field begins with the choice of a finite-dimensional module and with differential forms taking values in this module. The detailed relationship can be established using a parent approach which allows one to systematically construct an unfolded formulation starting from the BV-BRST formulation, respectively its BRST first quantized formulation for linear theories: the space where the $p$-form fields take values in the minimal unfolded formulation can then be shown to coincide with $H^{-p}(\Omega)$, and hence with order $p$ global reducibility parameters.

\section{Acknowledgements}

The work of GB is supported by the Fund for Scientific Research-FNRS (Belgium), by IISN-Belgium, and by "Communauté française de Belgique - Actions de Recherche Concertées". The research of XB and MG was supported by the Russian Science Foundation grant 14-42-00047 in association with Lebedev Physical Institute. XB is grateful to Sogang University for hospitality while this work was being completed. XB and MG would like to thank K.B. Alkalaev, E. Joung, R. Metsaev, Y. Nakayama, I. Tipunin, A. Tseytlin, M.A. Vasiliev, A. Verbovetsky and especially O. Shaynkman for valuable discussions.

Note added: While the present paper was in preparation, reference [107] appeared on arXiv, which also discusses 4-dimensional maximal-depth Fradkin-Tseytlin fields.

\section{References}

[1] A. Vinogradov, "On the algebra-geometric foundations of Lagrangian field theory," Sov. Math. Dokl. 18 (1977) 1200.

[2] A. Vinogradov, "A spectral sequence associated with a nonlinear differential equation and algebra-geometric foundations of Lagrangian field theory with constraints," Sov. Math. Dokl. 19 (1978) 144.

[3] A. Vinogradov, "The C-spectral sequence, Lagrangian formalism, and conservation laws. I. The linear theory. II. The non linear theory," J. Math. Anal. 100 (1984) 1.

[4] A. Vinogradov, Cohomological Analysis of Partial Differential Equations and Secondary Calculus, vol. 204 of Translations of Mathematical Monographs. AMS, 2001.

[5] I. Anderson, "Introduction to the variational bicomplex," in Mathematical Aspects of Classical Field Theory, M. Gotay, J. Marsden, and V. Moncrief, eds., vol. 132 of Contemporary Mathematics, pp. 51-73. Amer. Math. Soc., 1992. 
[6] L. Dickey, "Soliton Equations and Hamiltonian Systems," 1991.

[7] P. Olver, Applications of Lie Groups to Differential Equations. Spinger Verlag, New York, 2nd ed., 1993. 1st ed., 1986.

[8] I. Anderson, "The variational bicomplex," tech. rep., Formal Geometry and Mathematical Physics, Department of Mathematics, Utah State University, 1989.

[9] G. W. Bluman and S. C. Anco, Symmetry and integration methods for differential equations. Springer, 2002.

[10] G. W. Bluman, A. Cheviakov, and S. Anco, Applications of Symmetry Methods to Partial Differential Equations. Springer, 2009.

[11] J. Krasil'shchik and A. Verbovetsky, "Homological Methods in Equations of Mathematical Physics," 1998.

[12] S. C. Anco and J. Pohjanpelto, "Conserved Currents of Massless Fields of Spin s greater than 1/2," Proc.Roy.Soc.Lond. A459 (2003) 1215-1240, math-ph/0202019.

[13] J. Pohjanpelto and S. C. Anco, "Generalized Symmetries of Massless Free Fields on Minkowski Space," SIGMA 4 (2008) 004, 0801.1892.

[14] X. Bekaert and M. Grigoriev, "Manifestly conformal descriptions and higher symmetries of bosonic singletons," SIGMA 6 (2010) 038, 0907.3195.

[15] M. G. Eastwood, "Higher symmetries of the Laplacian,” Annals Math. 161 (2005) 1645-1665, hep-th/0206233.

[16] M. Fierz and W. Pauli, "On relativistic wave equations for particles of arbitrary spin in an electromagnetic field," Proc. Roy. Soc. Lond. A173 (1939) 211-232.

[17] C. Fronsdal, “Massless Fields with Integer Spin,” Phys.Rev. D18 (1978) 3624.

[18] J. Fang and C. Fronsdal, "Massless Fields with Half Integral Spin," Phys. Rev. D18 (1978) 3630.

[19] L. Gross, "Norm Invariance of Mass-Zero Equations under the Conformal Group," Journal of Mathematical Physics 5 (1964), no. 5, 687-695.

[20] A. J. Bracken and B. Jessup, "Local conformal invariance of the wave equation for finite component fields. I. The conditions for invariance, and fully reducible fields," J. Math. Phys. 23 (1982) 1925-1946.

[21] W. Siegel, "All free conformal representations in all dimensions," Int. J. Mod. Phys. A4 (1989) 2015.

[22] R. Metsaev, "All conformal invariant representations of d-dimensional anti-de Sitter group," Mod.Phys.Lett. A10 (1995) 1719-1731.

[23] E. Angelopoulos and M. Laoues, "Masslessness in n-dimensions," Rev. Math. Phys. 10 (1998) 271-300, hep-th/9806100

[24] R. Penrose, "Zero rest mass fields including gravitation: Asymptotic behavior," Proc. Roy. Soc. Lond. A284 (1965) 159.

[25] M. Eastwood and B. Rice, J.W., "Conformally invariant differential operators on Minkowski space and their curved analogues," Commun.Math.Phys. 109 (1987) 207-228.

[26] B. Dobrev, V.K., "Invariant differential operators for non-compact Lie groups: Parabolic subalgebras," Rev.Math.Phys. 20 (2008) 407-449, hep-th/0702152. 
[27] O. Shaynkman, I. Y. Tipunin, and M. Vasiliev, "Unfolded form of conformal equations in M dimensions and o(M + 2) modules," Rev.Math.Phys. 18 (2006) 823-886, hep-th/0401086.

[28] Y. Nakayama, "Scale invariance vs conformal invariance," Phys.Rept. 569 (2015) 1-93, 1302.0884 .

[29] A. Dymarsky and A. Zhiboedov, "Scale-invariant breaking of conformal symmetry," 1505.01152 .

[30] B. de Wit and D. Z. Freedman, "Systematics of Higher Spin Gauge Fields," Phys. Rev. D21 (1980) 358.

[31] I. Batalin and G. Vilkovisky, "Gauge Algebra and Quantization," Phys.Lett. B102 (1981) $27-31$.

[32] I. Batalin and G. Vilkovisky, "Feynman Rules For Reducible Gauge Theories," Phys.Lett. B120 (1983) 166-170.

[33] I. Batalin and G. Vilkovisky, "Closure of the Gauge Algebra, Generalized Lie Equations and Feynman Rules," Nucl.Phys. B234 (1984) 106-124.

[34] I. Batalin and G. Vilkovisky, "Existence Theorem for Gauge Algebra," J.Math.Phys. 26 (1985) 172-184.

[35] M. Henneaux and C. Teitelboim, Quantization of Gauge Systems. Princeton University Press, 1992.

[36] J. Gomis, J. París, and S. Samuel, "Antibracket, antifields and gauge theory quantization," Phys. Rept. 259 (1995) 1-145, hep-th/9412228.

[37] G. Barnich, F. Brandt, and M. Henneaux, "Local BRST cohomology in the antifield formalism. I. General theorems," Commun. Math. Phys. 174 (1995) 57-92, hep-th/9405109

[38] G. Barnich, F. Brandt, and M. Henneaux, "Local BRST cohomology in gauge theories," Phys.Rept. 338 (2000) 439-569, hep-th/ 0002245

[39] G. Barnich, "A Note on gauge systems from the point of view of Lie algebroids," AIP Conf.Proc. 1307 (2010) 7-18, 1010.0899.

[40] M. Henneaux, "Elimination of the Auxiliary Fields in the Antifield Formalism," Phys. Lett. B238 (1990) 299.

[41] G. Barnich and F. Brandt, "Covariant theory of asymptotic symmetries, conservation laws and central charges," Nucl. Phys. B633 (2002) 3-82, hep-th/0111246.

[42] A. Sen and B. Zwiebach, "A Note on gauge transformations in Batalin-Vilkovisky theory," Phys. Lett. B320 (1994) 29-35, hep-th/9309027.

[43] A. Nersessian and P. Damgaard, "Comments on the covariant $S p(2)$ symmetric Lagrangian BRST formalism," Phys.Lett. B355 (1995) 150-156, hep-th/9505009

[44] M. A. Grigoriev, A. M. Semikhatov, and I. Y. Tipunin, "Gauge symmetries of the master action in the Batalin- Vilkovisky formalism," J. Math. Phys. 40 (1999) 1792-1806, hep-th/9804156.

[45] G. Barnich, M. Grigoriev, A. Semikhatov, and I. Tipunin, "Parent field theory and unfolding in BRST first-quantized terms," Commun.Math.Phys. 260 (2005) 147-181, hep-th/0406192

[46] G. Barnich and M. Grigoriev, "First order parent formulation for generic gauge field theories," JHEP 01 (2011) 122, 1009.0190. 
[47] M. Bochicchio, "String Field Theory in the Siegel Gauge," Phys. Lett. B188 (1987) 330.

[48] M. Bochicchio, "Gauge Fixing for the Field Theory of the Bosonic String," Phys. Lett. B193 (1987) 31.

[49] C. B. Thorn, "Perturbation Theory for Quantized String Fields," Nucl. Phys. B287 (1987) 61.

[50] C. B. Thorn, "String Field Theory," Phys. Rept. 175 (1989) 1-101.

[51] G. Barnich and M. Grigoriev, "Hamiltonian BRST and Batalin-Vilkovisky formalisms for second quantization of gauge theories," Commun.Math.Phys. 254 (2005) 581-601, hep-th/0310083.

[52] G. Barnich and M. Grigoriev, "A Poincare lemma for sigma models of AKSZ type," J.Geom.Phys. 61 (2011) 663-674, 0905.0547

[53] M. Henneaux, "On The Algebraic Structure Of The BRST Symmetry," in Physics, Geometry, and Topology, H. Lee, ed., pp. 81-104, Banff Summer School in Theoretical Physics, NATO ASI 1989. Plenum Press, 1990.

[54] P. O. Kazinski, S. L. Lyakhovich, and A. A. Sharapov, "Lagrange structure and quantization," JHEP 07 (2005) 076, hep-th/0506093.

[55] D. S. Kaparulin, S. L. Lyakhovich, and A. A. Sharapov, "Rigid Symmetries and Conservation Laws in Non-Lagrangian Field Theory," J. Math. Phys. 51 (2010) 082902, 1001.0091 .

[56] D. Kaparulin, S. Lyakhovich, and A. Sharapov, "Lagrange Anchor and Characteristic Symmetries of Free Massless Fields," SIGMA 8 (2012) 021, 1112.1860.

[57] M. A. Vasiliev, "Equations of Motion of Interacting Massless Fields of All Spins as a Free Differential Algebra," Phys. Lett. B209 (1988) 491-497.

[58] V. E. Lopatin and M. A. Vasiliev, "Free Massless Bosonic Fields of Arbitrary Spin in d-dimensional De Sitter Space," Mod. Phys. Lett. A3 (1988) 257.

[59] K. Alkalaev, O. Shaynkman, and M. Vasiliev, "On the frame - like formulation of mixed symmetry massless fields in (A)dS(d)," Nucl.Phys. B692 (2004) 363-393, hep-th/0311164.

[60] M. A. Vasiliev, "Actions, charges and off-shell fields in the unfolded dynamics approach," Int. J. Geom. Meth. Mod. Phys. 3 (2006) 37-80, hep-th/ 0504090.

[61] E. Skvortsov, "Mixed-Symmetry Massless Fields in Minkowski space Unfolded," JHEP 0807 (2008) 004, 0801.2268 .

[62] M. Vasiliev, "Bosonic conformal higher-spin fields of any symmetry," Nucl.Phys. B829 (2010) 176-224, 0909.5226.

[63] G. Barnich and M. Grigoriev, "Parent form for higher spin fields on anti-de Sitter space," JHEP 08 (2006) 013, hep-th/0602166.

[64] M. Grigoriev, "Parent formulation at the Lagrangian level," JHEP 07 (2011) 061, 1012.1903 .

[65] M. Grigoriev and A. Waldron, "Massive Higher Spins from BRST and Tractors," Nucl. Phys. B853 (2011) 291-326, 1104.4994.

[66] M. Grigoriev, "Off-shell gauge fields from BRST quantization," hep-th/0605089.

[67] K. B. Alkalaev, M. Grigoriev, and I. Y. Tipunin, "Massless Poincare modules and gauge invariant equations," Nucl. Phys. B823 (2009) 509-545, 0811.3999. 
[68] K. B. Alkalaev and M. Grigoriev, "Unified BRST description of AdS gauge fields," Nucl. Phys. B835 (2010) 197-220, 0910.2690.

[69] X. Bekaert and M. Grigoriev, "Notes on the ambient approach to boundary values of AdS gauge fields," J.Phys. A46 (2013) 214008, 1207.3439.

[70] X. Bekaert and M. Grigoriev, "Higher order singletons, partially massless fields and their boundary values in the ambient approach," Nucl.Phys. B876 (2013) 667-714, 1305.0162 .

[71] V. Bargmann and E. P. Wigner, "Group Theoretical Discussion of Relativistic Wave Equations," Proc.Nat.Acad.Sci. 34 (1948) 211.

[72] S. Weinberg, "Photons and gravitons in perturbation theory: Derivation of Maxwell's and Einstein's equations," Phys. Rev. 138 (1965) B988-B1002.

[73] E. S. Fradkin and A. A. Tseytlin, "Conformal Supergravity," Phys. Rept. 119 (1985) 233-362.

[74] X. Bekaert and N. Boulanger, "Tensor gauge fields in arbitrary representations of GL(D,R): Duality and Poincare lemma," Commun. Math. Phys. 245 (2004) 27-67, hep-th/0208058.

[75] X. Bekaert and N. Boulanger, "On geometric equations and duality for free higher spins," Phys. Lett. B561 (2003) 183-190, hep-th/0301243.

[76] X. Bekaert, N. Boulanger, and D. Francia, "Mixed-symmetry multiplets and higher-spin curvatures," J.Phys. A48 (2015), no. 22, 225401, 1501.02462

[77] E. D. Skvortsov, "Frame-like Actions for Massless Mixed-Symmetry Fields in Minkowski space," 0807.0903

[78] E. Skvortsov, "Gauge fields in (A)dS(d) and Connections of its symmetry algebra," J.Phys. A42 (2009) 385401, 0904.2919.

[79] N. Boulanger, C. Iazeolla, and P. Sundell, "Unfolding Mixed-Symmetry Fields in AdS and the BMV Conjecture: I. General Formalism," JHEP 07 (2009) 013, 0812 . 3615.

[80] N. Boulanger, C. Iazeolla, and P. Sundell, "Unfolding Mixed-Symmetry Fields in AdS and the BMV Conjecture: II. Oscillator Realization,” JHEP 07 (2009) 014, 0812 . 4438.

[81] K. Alkalaev and M. Grigoriev, "Unified BRST approach to (partially) massless and massive AdS fields of arbitrary symmetry type," Nucl. Phys. B853 (2011) 663-687, 1105.6111 .

[82] S. Ouvry and J. Stern, "Gauge Fields of Any Spin and Symmetry," Phys. Lett. B177 (1986) 335.

[83] A. K. H. Bengtsson, "A One-dimensional Invariance Principle for Gauge Fields of Integer Spin,” Phys. Lett. B189 (1987) 337.

[84] M. Henneaux and C. Teitelboim, First and second quantized point particles of any spin, ch. 9, pp. 113-152. Quantum mechanics of fundamental systems 2, Centro de Estudios Científicos de Santiago. Plenum Press, 1987.

[85] A. Sagnotti and M. Tsulaia, "On higher spins and the tensionless limit of string theory," Nucl. Phys. B682 (2004) 83-116, hep-th/0311257

[86] G. Barnich, G. Bonelli, and M. Grigoriev, "From BRST to light-cone description of higher spin gauge fields," hep-th/0502232.

[87] M. A. Vasiliev, "Nonlinear equations for symmetric massless higher spin fields in (A)dS(d)," Phys. Lett. B567 (2003) 139-151, hep-th/0304049. 
[88] G. Barnich, N. Bouatta, and M. Grigoriev, "Surface charges and dynamical Killing tensors for higher spin gauge fields in constant curvature spaces," JHEP 10 (2005) 010, hep-th/0507138.

[89] G. Mack and A. Salam, "Finite component field representations of the conformal group," Annals Phys. 53 (1969) 174-202.

[90] J. Labastida and T. Morris, "Massless Mixed Symmetry Bosonic Free Fields," Phys.Lett. B180 (1986) 101.

[91] J. M. F. Labastida, "Massless Particles in Arbitrary Representations of the Lorentz Group," Nucl. Phys. B322 (1989) 185.

[92] C. Burdik, A. Pashnev, and M. Tsulaia, "On the mixed symmetry irreducible representations of the Poincare group in the BRST approach," Mod. Phys. Lett. A16 (2001) 731-746, hep-th/0101201

[93] A. Campoleoni, D. Francia, J. Mourad, and A. Sagnotti, "Unconstrained Higher Spins of Mixed Symmetry. II. Fermi Fields,” Nucl.Phys. B828 (2010) 405-514, 0904.4447.

[94] E. Skvortsov and Y. Zinoviev, "Frame-like Actions for Massless Mixed-Symmetry Fields in Minkowski space. Fermions," Nucl.Phys. B843 (2011) 559-569, 1007.4944.

[95] S. Deser and R. I. Nepomechie, "Anomalous Propagation of Gauge Fields in Conformally Flat Spaces," Phys. Lett. B132 (1983) 321.

[96] S. Deser and R. I. Nepomechie, "Gauge Invariance Versus Masslessness in De Sitter Space," Annals Phys. 154 (1984) 396.

[97] S. Deser and A. Waldron, "Partial masslessness of higher spins in (A)dS," Nucl.Phys. B607 (2001) 577-604, hep-th/0103198

[98] S. Deser and A. Waldron, "Gauge invariances and phases of massive higher spins in (A)dS," Phys.Rev.Lett. 87 (2001) 031601, hep-th/0102166.

[99] E. D. Skvortsov and M. A. Vasiliev, "Geometric formulation for partially massless fields," Nucl. Phys. $B 756$ (2006) 117-147, hep-th/0601095

[100] E. Joung, L. Lopez, and M. Taronna, "On the cubic interactions of massive and partially-massless higher spins in (A)dS," JHEP 1207 (2012) 041, 1203.6578.

[101] J. Erdmenger and H. Osborn, "Conformally covariant differential operators: Symmetric tensor fields," Class.Quant.Grav. 15 (1998) 273-280, gr-qc/97080 40.

[102] S. Deser and A. Waldron, "Partially Massless Spin 2 Electrodynamics," Phys.Rev. D74 (2006) 084036, hep-th/0609113

[103] S. Deser and A. Waldron, "Conformal invariance of partially massless higher spins," Phys.Lett. B603 (2004) 30, hep-th/ 0408155.

[104] R. R. Metsaev, "Massless mixed symmetry bosonic free fields in d- dimensional anti-de Sitter space-time," Phys. Lett. B354 (1995) 78-84.

[105] E. D. Skvortsov, "Gauge fields in (A)dS within the unfolded approach: algebraic aspects," JHEP 01 (2010) 106, 0910.3334.

[106] M. Vasiliev, "Conformal higher spin symmetries of 4-d massless supermultiplets and osp(L,2M) invariant equations in generalized (super)space," Phys.Rev. D66 (2002) 066006, hep-th/0106149

[107] M. Beccaria and A. A. Tseytlin, "On higher spin partition functions," J. Phys. A48 (2015), no. 27, 275401, 1503.08143. 